\title{
BacHBerry: BACterial Hosts for production of Bioactive phenolics from bERRY fruits
}

\author{
Alexey Dudnik (D. Filipa Almeida - Ricardo Andrade - Barbara Avila • \\ Pilar Bañados $\cdot$ Diane Barbay $\cdot$ Jean-Etienne Bassard $(\mathbb{D} \cdot$ \\ Mounir Benkoulouche $\cdot$ Michael Bott $₫$ - Adelaide Braga $\cdot$ Dario Breitel • \\ Rex Brennan · Laurent Bulteau • Celine Chanforan • Inês Costa •

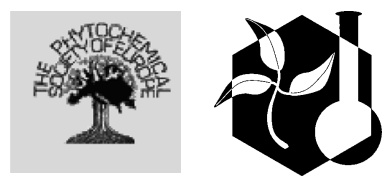 \\ Rafael S. Costa $\cdot$ Mahdi Doostmohammadi $\cdot$ Nuno Faria $\cdot$ Chengyong Feng \\ Armando Fernandes $\cdot$ Patricia Ferreira $\cdot$ Roberto Ferro $\cdot$ Alexandre Foito $\cdot$ \\ Sabine Freitag • Gonçalo Garcia • Paula Gaspar • Joana Godinho-Pereira • \\ Björn Hamberger • András Hartmann · Harald Heider • Carolina Jardim • \\ Alice Julien-Laferriere • Nicolai Kallscheuer $(\mathbb{D} \cdot$ Wolfgang Kerbe • \\ Oscar P. Kuipers $(\mathbb{D} \cdot$ Shanshan Li $\cdot$ Nicola Love $\cdot$ Alberto Marchetti-Spaccamela \\ Jan Marienhagen $(\mathbb{D} \cdot$ Cathie Martin • Arnaud Mary • Vincent Mazurek • \\ Camillo Meinhart • David Méndez Sevillano • Regina Menezes • \\ Michael Naesby • Morten H. H. Nørholm • Finn T. Okkels • Joana Oliveira • \\ Marcel Ottens · Delphine Parrot - Lei Pei - Isabel Rocha - Rita Rosado-Ramos • \\ Caroline Rousseau • Marie-France Sagot • Claudia Nunes dos Santos • \\ Markus Schmidt • Tatiana Shelenga - Louise Shepherd • Ana Rita Silva • \\ Marcelo Henriques da Silva • Olivier Simon - Steen Gustav Stahlhut • \\ Ana Solopova $\cdot$ Artem Sorokin · Derek Stewart • Leen Stougie • \\ Shang Su • Vera Thole Olga Tikhonova $\cdot$ Martin Trick $\cdot$ Philippe Vain • \\ André Veríssimo $\cdot$ Ana Vila-Santa $\cdot$ Susana Vinga $\cdot$ Michael Vogt $\cdot$ \\ Liangsheng Wang - Lijin Wang - Wei Wei - Sandra Youssef - Ana Rute Neves • \\ Jochen Forster $\mathbb{1 0}$
}

This article is written by the BacHBerry consortium (www. bachberry.eu) and represents the collective effort of all participating institutions. The authors are therefore listed in alphabetical order.

Electronic supplementary material The online version of this article (doi:10.1007/s11101-017-9532-2) contains supplementary material, which is available to authorized users.

A. Dudnik $(\bowtie) \cdot$ R. Ferro · P. Gaspar ·

M. H. H. Nørholm · S. G. Stahlhut · J. Forster

The Novo Nordisk Foundation Center for

Biosustainability, Technical University of Denmark,

Kemitorvet, Building 220, 2800 Kgs., Lyngby, Denmark

e-mail: adud@biosustain.dtu.dk
Abstract BACterial Hosts for production of Bioac-

tive phenolics from bERRY fruits (BacHBerry) was a 3-year project funded by the Seventh Framework Programme (FP7) of the European Union that ran between November 2013 and October 2016. The overall aim of the project was to establish a sustainable and economically-feasible strategy for the production

\footnotetext{
R. Andrade - L. Bulteau · A. Julien-Laferriere ·

A. Marchetti-Spaccamela · A. Mary .

D. Parrot · M.-F. Sagot - L. Stougie

Institut National de Recherche en Informatique et Automatique, 43 Boulevard du 11 Novembre 1918, 69622 Villeurbanne Cedex, France
} 
of novel high-value phenolic compounds isolated from berry fruits using bacterial platforms. The project aimed at covering all stages of the discovery and precommercialization process, including berry collection, screening and characterization of their bioactive components, identification and functional characterization of the corresponding biosynthetic pathways, and construction of Gram-positive bacterial cell factories producing phenolic compounds. Further activities included optimization of polyphenol extraction methods from bacterial cultures, scale-up of production by fermentation up to pilot scale, as well as societal and economic analyses of the processes. This review article summarizes some of the key findings obtained throughout the duration of the project.

Keywords Berries - Bioprospecting - Microbial cell factories $\cdot$ Polyphenols $\cdot$ Sustainable production

\section{Abbreviations \\ 4CL 4-coumaryl-CoA ligase \\ AD Alzheimer's disease \\ ANS Anthocyanidin synthase \\ ALS Amyotrophic lateral sclerosis; anthocyanidin synthase \\ ANR Anthocyanidin reductase}

\section{B. Avila $\cdot$ P. Bañados}

Facultad De Agronomía e Ingeniería Forestal, Pontificia Universidad Católica de Chile, Av. Vicuña Mackenna Ote, 4860 Macul, Chile

D. Barbay - M. Benkoulouche $\cdot$ H. Heider .

M. Naesby - C. Rousseau

Evolva, Duggingerstrasse 23, 4053 Reinach, Switzerland

J.-E. Bassard · B. Hamberger

Department of Plant and Environmental Science, University of Copenhagen, 1871 Frederiksberg, Denmark

M. Bott · N. Kallscheuer · J. Marienhagen · M. Vogt Forschungszentrum Jülich $\mathrm{GmbH}$, Institut für Bio- und Geowissenschaften, IBG-1: Biotechnologie, WilhelmJohnen-Straße, 52428 Jülich, Germany

A. Braga $\cdot$ N. Faria $\cdot$ P. Ferreira $\cdot$ J. Oliveira

I. Rocha $\cdot$ A. R. Silva

Biotempo, Avepark - Edif. Spinpark, Zona Industrial da Gandra, Barco, 4805-017 Guimarães, Portugal
$\mathrm{C}^{\prime} \mathrm{H} \quad p$-coumaroyl shikimate/quinate $3^{\prime}$ hydroxylase

$\mathrm{C} 4 \mathrm{H} \quad$ Cinnamate 4-hydroxylase

CBD Convention on biological diversity

CaN Calcineurin

CDRE Calcineurin-dependent responsive element

CHI Chalcone isomerase

CHR Chalcone reductase

CHS Chalcone synthase

CPR Cytochrome-P450 reductase

CVs Column volumes

CYP Cytochromes P450

DFR Dihydroflavonol 4-reductase

F3H Flavanone 3-hydroxylase

$\mathrm{F}^{\prime} \mathrm{H} \quad$ Flavonoid $3^{\prime}$-hydroxylase

$\mathrm{F}^{\prime} 5^{\prime} \mathrm{H}$ Flavonoid $3^{\prime}-5^{\prime}$-hydroxylase

FLS Flavonol synthase

FNS Flavone synthase

FP7 European Commission's the Seventh

Framework programme

GFP Green fluorescent protein

GRAS Generally regarded as safe

HCT Hydroxycinnamoyl-CoA shikimate/quinate hydroxycinnamoyltransferase

HD Huntington's disease

HPLC High-performance liquid chromatography

LAR Leucoanthocyanidin reductase

C. Chanforan · V. Mazurek · F. T. Okkels · O. Simon Chr. Hansen Natural Colors A/S, Agern Alle 24, 2970 Hørsholm, Denmark

R. S. Costa - M. Doostmohammadi - A. Fernandes · A. Hartmann - A. Veríssimo $\cdot$ A. Vila-Santa $\cdot$ S. Vinga IDMEC, Instituto Superior Técnico, Universidade de Lisboa, Av. Rovisco Pais 1, 1049-001 Lisbon, Portugal

R. Brennan · A. Foito - S. Freitag · L. Shepherd .

D. Stewart

The James Hutton Institute, Invergowrie, DD2 5DA, Dundee, Scotland, UK

O. P. Kuipers · A. Solopova

Groningen Biomolecular Sciences and Biotechnology Institute, Department of Molecular Genetics, University of Groningen, Linnaeusborg, Nijenborgh 7,

9747 AG Groningen, The Netherlands

D. Breitel · N. Love · C. Martin · V. Thole .

M. Trick · P. Vain

John Innes Centre, Norwich Research Park,

NR4 7UH Norwich, UK 
LC- Liquid-chromatography mass-spectrometry

MS

NDs Neurodegenerative diseases

NFAT Nuclear Factor of Activated T-cells

NFкB Nuclear Factor $\kappa B$

NP- The Nagoya protocol on access to genetic

ABS resources and the fair and equitable sharing of benefits arising from their utilization

OMT $O$-methyltransferase

PAL Phenylalanine ammonia-lyase

PCA Principal component analysis

PD Parkinson's disease

PLS Partial least squares

RNA- RNA sequencing technology

seq

STS Stilbene synthase

SVR Support vector regression

TAL Tyrosine ammonia-lyase

UFGT Flavonoid 3-O-glucosyltransferase eu) has received funding from the European Commission's Seventh Framework Programme (FP7) under the theme KBBE.2013.3.1-01: "Plant High Value Products-from discovery to final product" with the goal of bringing together experts from the fields of plant biology, industrial biotechnology, analytical chemistry, and social sciences in order to assemble and validate a complete pipeline for discovery and microbial production of novel phenolic compounds of plant origin. The consortium consisted of twelve academic groups, five small and medium sized enterprises and one large enterprise from eleven different countries, namely Austria, Chile, China, Denmark, France, Germany, The Netherlands, Portugal, Russia, Switzerland and the United Kingdom. The diversity of partners has allowed access to a variety of expertise and to plant material that had not been characterized extensively.

\section{Polyphenols as a source of bioactive molecules}

\section{Introduction}

BacHBerry (BACterial Hosts for production of Bioactive phenolics from bERRY fruits, www.bachberry.

W. Kerbe · C. Meinhart - L. Pei · M. Schmidt .

S. Youssef

Biofaction KG, Kundmanngasse 39/12, 1030 Vienna,

Austria

D. M. Sevillano $\cdot$ M. Ottens · M. H. da Silva

Department of Biotechnology, Delft University of

Technology, van der Maasweg 9, 2629 HZ Delft, The

Netherlands

A. F. Almeida - I. Costa - G. Garcia

J. Godinho-Pereira · C. Jardim · R. Menezes ·

R. Rosado-Ramos - C. N. dos Santos

iBET, Instituto de Biologia Experimental e Tecnológica, Apartado 12, 2780-901 Oeiras, Portugal

T. Shelenga $\cdot$ A. Sorokin $\cdot$ O. Tikhonova

Fruit Crops Genetic Resources Department, N. I. Vavilov Research Institute of Plant Industry, B. Morskaya Street 42-44, 190000 St. Petersburg, Russia

C. Feng · S. Li · S. Su - L. Wang - L. Wang - W. Wei Institute of Botany, The Chinese Academy of Sciences, 20 Nanxincun, Xiangshan, 100093 Beijing, China

A. R. Neves

Chr. Hansen Holding A/S, Bøge Alle 10-12,

2970 Hørsholm, Denmark
Historically, plants have been not only important sources of nutrients and energy in the human diet but also have provided the fundamentals of traditional and

D. Stewart

Institute of Mechanical, Process and Energy Engineering, School of Engineering and Physical Sciences, Heriot Watt University, Edinburgh, Scotland, UK

A. F. Almeida · I. Costa - G. Garcia .

J. Godinho-Pereira · C. Jardim · R. Menezes ·

R. Rosado-Ramos · C. N. dos Santos

Instituto de Tecnologia Química e Biológica António

Xavier, Universidade Nova de Lisboa, Av. da República,

2780-157 Oeiras, Portugal

A. Braga - N. Faria $\cdot$ P. Ferreira · J. Oliveira .

Centre of Biological Engineering, University of Minho,

Campus de Gualtar, 4710-057 Braga, Portugal

M. Doostmohammadi · L. Stougie

School of Mathematics, University of Edinburgh,

EH9 3FD Edinburgh, Scotland, UK

L. Stougie

Centrum Wiskunde \& Informatica, Science Park 123, 1098 XG Amsterdam, Netherlands
I. Rocha · A. R. Silva 
modern medicine. The Plantae kingdom has been estimated to produce more than 200,000 different chemical compounds (Weckwerth 2003), including compounds with proven and potential medical applications. This is reflected in the high number of pharmaceutical products based on, or derived from, plant natural products, such as aspirin, morphine, quinine, paclitaxel and artemisinin (Cragg and Newman 2013). Among the different classes of specialized (i.e. secondary) metabolites, phenylpropanoids stand out due to their diversity and ubiquity in the plant kingdom. Fleshy fruits, a rich source of bioactive components, such as ascorbate (vitamin C), terpenoids and polyphenols, are considered to have one the highest antioxidant capacity of commonly eaten foods (Halvorsen et al. 2006). Polyphenol content and composition can vary substantially between fleshyfruit varieties (Carvalho et al. 2013; Miladinović et al. 2014; Kula et al. 2016), different cultivation conditions (Josuttis et al. 2013; Vagiri et al. 2013; Mazur et al. 2014), harvest times and as a result of different post-harvest factors (Kårlund et al. 2014; Stavang et al. 2015), but particularly between species (Moyer et al. 2001; McDougall et al. 2008; Ruiz et al. 2010; Marhuenda et al. 2016). Furthermore, plant polyphenols are increasingly being associated with putative bioactivities offering protection against several cardiovascular (Goszcz et al. 2015) and neurological diseases (Figueira et al. 2017).

The hydroxystilbenes are a group of polyphenols with a $\mathrm{C}_{6}-\mathrm{C}_{2}-\mathrm{C}_{6}$ skeleton with two or more hydroxyl groups, where a central carbon-carbon double bond is conjugated with two phenolic moieties (Quideau et al. 2011; Kasiotis et al. 2013). The most well-known representative of this group is resveratrol (trans-3,5,4'hydroxystilbene; Fig. 1a). The compound has gained increasing attention with the discovery of the "French paradox", an observation that despite a diet with relatively high amounts of saturated fats, French people suffer a relatively low incidence of mortality from coronary heart disease (Renaud and de Lorgeril 1992; Catalgol et al. 2012). Like other polyphenols, stilbenes can be further decorated by $O$-methylation (e.g. resulting in 3-methoxy-resveratrol, or pinostilbene, and 3,5'-dimethoxy-resveratrol, or pterostilbene), acylation (hydrangenic acid) or by glycosylation (e.g. piceid and resveratroloside; Kim et al. 2002; Becker et al. 2003; Zhang et al. 2009; Wang et al. 2015).

The flavonoids are one of the largest families of phenolic compounds. Flavonoids are characterized by their basic skeleton composed of three rings (Ghosh and Scheepens 2009), including two benzene rings (A $\&$ B) and one heterocyclic ring (C; Fig. 1b). So far, over 5000 naturally occurring flavonoids have been characterized from various plants and they have been classified into six subgroups, including anthocyanidins (e.g. cyanidin and delphinidin); flavan-3-ols (e.g. catechin and epicatechin); flavonols (e.g. quercetin and kaempferol); flavones (e.g. luteonin and apigenin); isoflavones (e.g. genistein and daidzein), and flavanones (e.g. hesperetin and naringenin).

Within each subgroup, considerable variation can exist when it comes to phenolic composition of different fruits, and in particular of berries. Anthocyanin composition provides an excellent example of this diversity: the red-orange color of strawberries is due to the presence of pelargonidin-type anthocyanins in the flesh and skin, whereas the deep purple-black color of blackcurrants is due to the accumulation of high amounts of delphinidin and cyanidin-type anthocyanins in the skin.

Anthocyanidins are flavonoids, which are characterized by a hydroxyl group in position 3 and a C-ring with a positively-charged oxonium ion (Fig. 1b). Anthocyanins are water-soluble glycosides of anthocyanidins, in which sugars, consisting of one or more hexoses, are linked to the 3-hydroxyl group of the
Fig. 1 a The molecular structure of the stilbene trans-resveratrol. b Basic flavonoid structure. $\mathrm{R}_{\mathrm{x}}$ are positions where decorating groups (e.g. hydroxyl, acyl, glycosyl, etc.), which differentiate the compounds and alter their chemical properties, could be attached
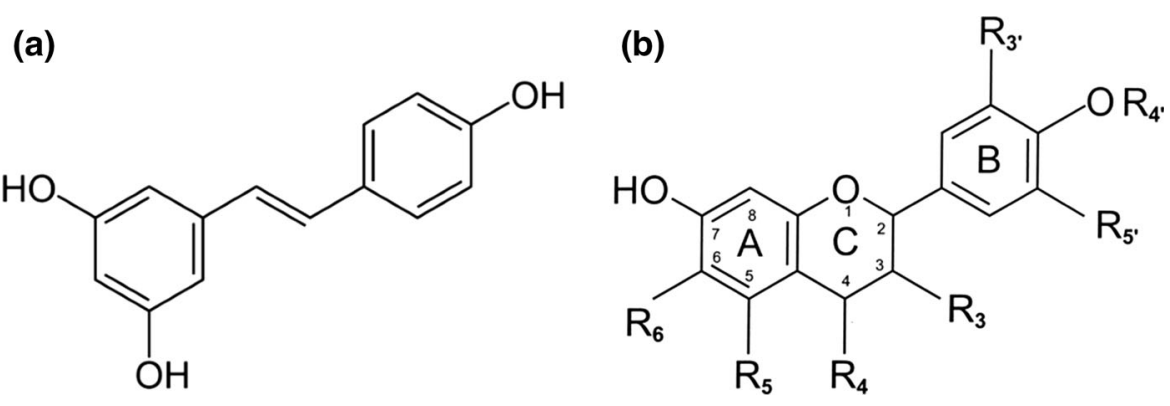
pyrylium ring (Heldt and Piechulla 2011). These compounds are responsible for the orange/red-to-blue coloration observed in some plants. The most common anthocyanidins include cyanidin (red), delphinidin (bluish-purple), malvinidin (reddish-purple), pelargonidin (orange-red), peonidin (pink) and petunidin (purple). The distribution of anthocyanidins can vary greatly among different berry species (Table 1).

Flavonoid compounds frequently are decorated with sugar moieties, sugar-acyl moieties (Giusti and Wrolstad 2003) and can be associated with other flavonoids. The patterns of decoration differ greatly amongst species (see supplementary Tables S1 and S2).

Flavonoids are far more prevalent and diverse in berry species than in other common fruits and vegetables. High flavonol contents are observed in cranberry, lingonberry and blackcurrant (Häkkinen et al. 1999), anthocyanins are the most abundant polyphenol pigments (2-5 $\mathrm{g} \mathrm{kg}^{-1}$ fresh weight) in berries (Määttä et al. 2001), and many simple phenolic acids are abundant in a wide range of berry species (Herrmann and Nagel 1989). Additionally, berries constitute one the most important dietary sources of ellagitannins, such as sanguiin H6 and lambertianin C (Törrönen 2009; Landete 2011), as well as condensed tannins, such as the proanthocyanidins (Rasmussen et al. 2005; Hellström et al. 2009). Stilbenes, in contrast, are not that widespread in berries: resveratrol shows highest abundance in grapes (up to 20-30 mg kg-1 fresh weight), but small amounts of resveratrol, pterostilbene and piceatannol have been also detected in blueberry, cowberry, cranberry, deerberry and lingonberry (Jeandet et al. 1991; Paredes-López et al. 2010; Manganaris et al. 2014). It is clear that soft-fruit species contain a staggering diversity of distinct polyphenol derivatives whose potential is yet to be harnessed.

The market for polyphenolic compounds has seen substantial growth over the past few years, and is expected to exceed 850 million USD by 2018 (Aranaz et al. 2016). The major factors contributing to this trend are the growing consumer awareness regarding the benefits of polyphenol consumption and the increasing use of polyphenol-containing extracts in food, beverage, and cosmetics products particularly in Asia (Jain and Ramawat 2013; Aranaz et al. 2016; Grand View Research Inc. 2016). The increase in demand also requires efficient and eco-friendly production processes, to improve on current manufacturing practices that mostly rely on extraction from various plant sources (e.g. roots, leaves or fruits) via complex

Table 1 Anthocyanidin structures (aglycone form) found in different berry species

\begin{tabular}{|c|c|c|c|c|c|c|}
\hline Type & Pelargonidin & Cyanidin & Delphinidin & Peonidin & Petunidin & Malvinidin \\
\hline \multicolumn{7}{|l|}{ Structure } \\
\hline Molecular formula & $\mathrm{C}_{15} \mathrm{H}_{11} \mathrm{O}_{5}^{+}$ & $\mathrm{C}_{15} \mathrm{H}_{11} \mathrm{O}_{6}{ }^{+}$ & $\mathrm{C}_{15} \mathrm{H}_{11} \mathrm{O}_{7}^{+}$ & $\mathrm{C}_{16} \mathrm{H}_{13} \mathrm{O}_{6}{ }^{+}$ & $\mathrm{C}_{16} \mathrm{H}_{13} \mathrm{O}_{7}^{+}$ & $\mathrm{C}_{17} \mathrm{H}_{15} \mathrm{O}_{7}^{+}$ \\
\hline Fragaria $^{\mathrm{a}}$ & + & + & - & - & - & - \\
\hline Ribes $^{\mathrm{a}}$ & + & + & + & - & + & - \\
\hline Rubua ${ }^{\mathrm{a}}$ & + & + & - & - & - & - \\
\hline Vaccinium $^{\mathrm{a}}$ & - & + & + & + & + & + \\
\hline $\operatorname{Vitis}^{\mathrm{a}}$ & - & + & + & + & + & + \\
\hline Lonicera $^{\mathrm{b}, \mathrm{e}}$ & + & + & + & + & - & + \\
\hline Lycium $^{\mathrm{c}}$ & - & - & + & - & + & + \\
\hline Berberis $^{\mathrm{d}}$ & - & + & + & + & + & + \\
\hline$U g n i^{\mathrm{d}}$ & - & + & - & + & - & - \\
\hline Aristotelia $^{\mathrm{f}}$ & - & + & + & - & - & - \\
\hline
\end{tabular}

The presence of a compound is noted with + whereas the absence is noted with

${ }^{\mathrm{a}}$ Phenol explorer database (http://phenol-explorer.eu), ${ }^{\mathrm{b}}$ (Chaovanalikit et al. 2004), ${ }^{\mathrm{c}}$ (Zheng et al. 2011), ${ }^{\mathrm{d}}$ (Ruiz et al. 2010), ${ }^{\text {e }}$ (Wang et al. 2016b), ${ }^{\mathrm{f}}$ (Fredes et al. 2014) 
downstream processing (Wang et al. 2016a). The BacHBerry consortium decided to address these challenges and set the following objectives: (1) to systematically analyze the phenolic contents in the large berry germplasm collections available from consortium members, (2) to establish a publicly-available database of transcriptomic and metabolic data obtained from berry bioprospecting within, as well as outside of the project, (3) to discover novel bioactivities in berry extracts against a range of human pathologies, such as Alzheimer's disease and Amyotrophic Lateral Sclerosis (ALS), by high-throughput screening with subsequent identification of functional biomolecules, (4) to identify the corresponding biosynthetic genes and perform functional characterization of the respective gene products, (5) to assay a selection of the biosynthetic genes for functionality in Gram-positive bacterial hosts and use those to construct bacterial cell factories for phenolic production, (6) to improve the production efficiency further by introducing modifications to the host metabolic networks predicted via rational design or computational tools developed within the project, (7) to design and optimize costeffective food-grade methods for extraction of phenolic compounds from bacterial fermentation broth, and (8) to optimize fermentation conditions and subsequently upscale the production to pilot plant levels.

The project commenced with the selection, sampling and whole-metabolite profiling of berries. In order to explore the potential of the phytochemical diversity present in different berry species, we undertook an untargeted characterization of a wide collection of berries from different species/cultivars utilizing metabolomics-based methods to aid the selection of candidate berry extracts for bioactivity screening.

\section{Phytochemical diversity in sampled berries}

Although significant advances have been made in characterizing the polyphenolic complement of berries, particularly in the context of cultivated species and genotypes, there is limited literature available regarding the phytochemical composition of wild and underutilized species/cultivars. Indeed, wild berries are commonly reported as particularly rich in diverse phenolic compounds, often as a result of phenolicassociated astringency having been bred out of cultivated species (Häkkinen et al. 1999). The phenolic diversity of individual berries has been highlighted in many publications (Määttä et al. 2003; Zadernowski et al. 2005; Milivojević et al. 2011), but studies that capture this broad diversity systematically are limited. In BacHBerry, we aimed not only to address this knowledge gap but also to go beyond the state-of-the-art by combining analyses of phenolic diversity with functional testing of berry extracts. The genera targeted in this study included Rubus, Ribes, Vaccinium, Lonicera, Lycium, Aristotelia, Berberis and Ugni collected from different locations in the world including Chile, China, Portugal, Russia and United Kingdom (see Supplementary Table S3).

\section{Berry extract metabolite profiling}

Fruit samples from a total of 112 species/genotypes were collected, extracted, and subsequently subjected to liquid-chromatography mass-spectrometry (LCMS) metabolomic analysis (see details in Supplementary Materials S1). An untargeted method was used to generate a total of 1890 mass spectral features (1506 and 384 in positive and negative modes, respectively), which were integrated to generate the dataset used for statistical analysis. Principal component analysis (PCA) was used as a multivariate statistical analysis tool to identify those species, which had the most distinct phytochemical profiles (Fig. 2a, b).

Using the four principal components it was possible to select the species/genotypes, which represented the broadest phenolic diversity (Table 2). In addition to selecting the outlier groups of species, it was also decided to include a small subset of samples (two Rubus idaeus genotypes), which did not separate from the majority of samples in the first principal components. This provided samples with phenolic profiles distinct from the outlier samples and standards for comparison between uncultivated/underutilized species and commonly grown species; in essence a commercial control. The fruits from the selected species (Table 2) were extracted and tested for bioactivity in baker's yeast (Saccharomyces cerevisiae) models for pathological processes associated with several chronic disorders.

\section{Berry extract bioactivity screening}

Evidence for the protective role of polyphenols against chronic diseases has increased over the past 20 years 
(a)

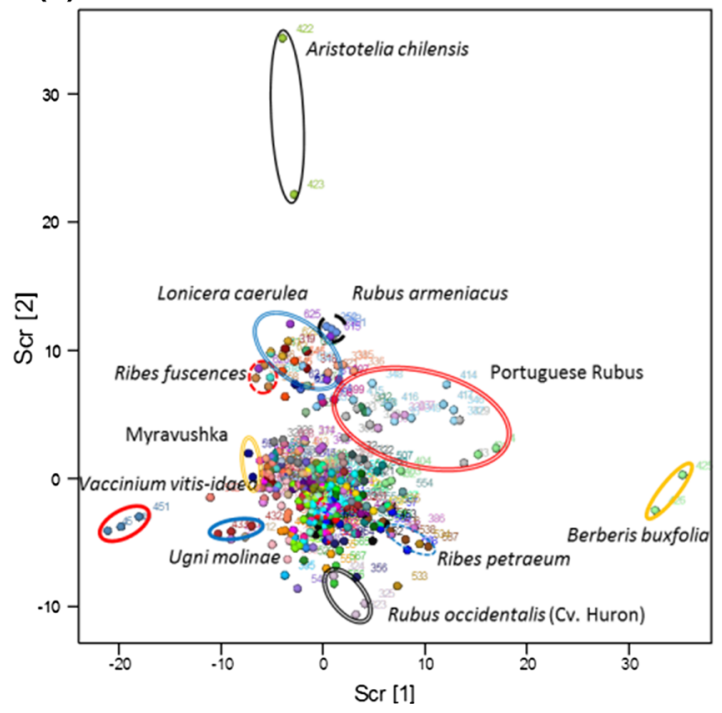

Fig. 2 Principal component analysis (PCA) plots of the full dataset without reference material and blanks. Each dot represents the profile of one sample aliquot whereas lines delimit groups of samples that cluster together. a PCA plots of the full dataset without reference material and blanks in positive

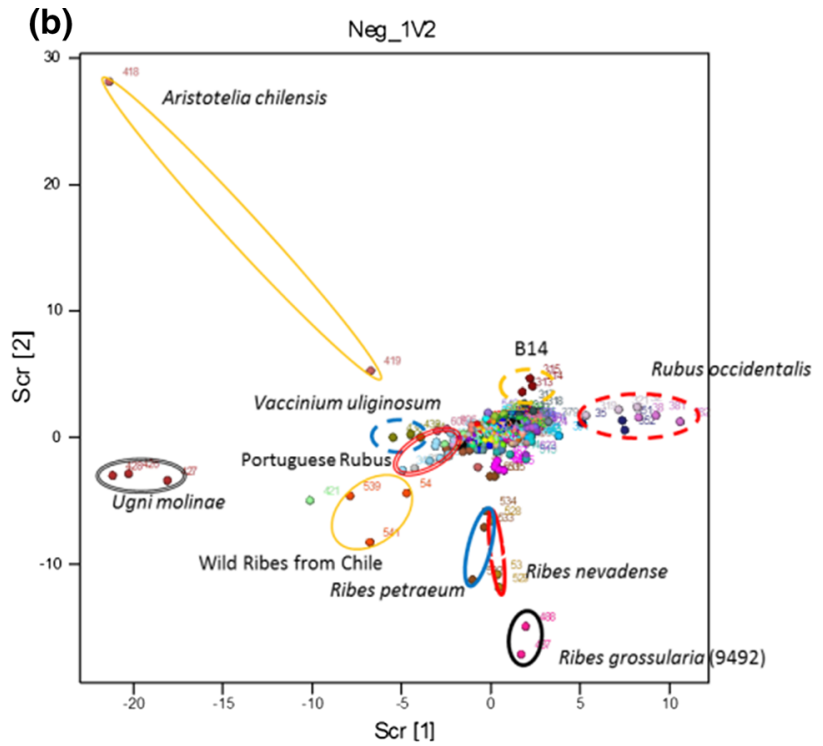

mode. Components (Scr) 1 and 2 explain 9.79 and $7.59 \%$ of the variation respectively. b PCA plots of the full dataset without reference material and blanks in negative mode. Components (Scr) 1 and 2 explain 13.20 and $10.30 \%$ of the variation respectively

Table 2 Selected species for bioactivity testing according to the phytochemical diversity

\begin{tabular}{|c|c|c|c|}
\hline Species & Variety/line & Species & Variety/line \\
\hline Aristotelia chilensis (Molina)Stuntz & - & Rubus armeniacus Focke & Himalayan Giant \\
\hline Berberis buxfolia Lam. & - & Rubus occidentalis L. & Huron \\
\hline Lonicera caerulea $\mathrm{L}$. & S322-23 & Rubus loganobaccus L.H.Bailey & Sunberry \\
\hline Lycium chinense Mill. & - & Rubus loganobaccus L.H.Bailey & Tayberry \\
\hline Ribes grossularia L. & 9294 & Rubus bartonii Newton & Ashton Cross \\
\hline Ribes grossularoides Maxim. & H1-12-1 & Rubus fruticosus L. agg. & Fantasia \\
\hline Ribes nevadense Kellog & - & Rubus brigantinus Samp. & - \\
\hline Ribes spp. & 644217 & Rubus genevierii Boreau. & - \\
\hline Ribes spp. & 1126 & Rubus henriquesii Samp. & - \\
\hline Ribes spp. & $2292-1$ & Rubus hochstetterorum Seub. & - \\
\hline Ribes spp. & Muravushka & Rubus sampaioanus Sudre ex Samp. & - \\
\hline Ribes spp. & - & Rubus vagabundus Samp. & - \\
\hline Ribes petraeum Wulf. & $\mathrm{R} 2-1-2$ & Rubus idaeus L. & Octavia \\
\hline Ribes x saundersii Jancz. & XB4 & Rubus idaeus L. & Prestige \\
\hline Ribes bethmontii Jancz. & XW6 & Ugni molinae Turcz. & Red Pearl \\
\hline Ribes rubrum L. & S11-3-36 & Vaccinium uliginosum L. & - \\
\hline Rubus spp. & B14 & Vaccinium vitis-idaea $\mathrm{L}$. & - \\
\hline
\end{tabular}


(Figueira et al. 2017; Goszcz et al. 2015). Neurodegenerative diseases (NDs) represent a group of chronic neurological conditions affecting millions of people worldwide, among which are the Alzheimer's Disease $(\mathrm{AD})$, the Parkinson's Disease (PD), the Huntington's Disease (HD), and Amyotrophic Lateral Sclerosis (ALS). All these diseases have in common the aggregation and deposition of protein aggregates, namely of amyloid $\beta 42$ (A $\beta 42$; O'Brien and Wong 2011), aSynuclenin ( $\alpha$ Syn; Shults 2006), huntingtin (HTT; Miller-Fleming et al. 2008) and FUsed in Sarcoma (FUS; Kwiatkowski et al. 2009; Vance et al. 2009), which represent the pathological hallmarks of $\mathrm{AD}, \mathrm{PD}, \mathrm{HD}$ and ALS, respectively. In addition, chronic activation of innate immune responses is a process closely associated with neurodegeneration. Its modulation is driven by persistent activation of key transcription factors, such as the Nuclear Factor of Activated T-cells (NFAT) and Nuclear Factor $\kappa \mathrm{B}$ (NFkB), which upregulate pro-inflammatory responses creating a positive feedback loop further amplifying initial stimuli. It has been argued that disruption of this loop may represent an important strategy to mitigate the progression of NDs. The pleiotropic effects of polyphenols have been shown to interfere with aggregation-driven neurodegeneration as well as to attenuate chronic inflammatory processes, thereby improving health of cellular and animal models (Figueira et al. 2017). Consequently, polyphenol-based therapies represent an underexplored strategy to minimize the huge social and economic impact of NDs.

The high degree of evolutionary conservation of fundamental biological processes among eukaryotes has established baker's yeast as a validated model organism to decipher the intricacies of human pathologies, particularly NDs, to identify molecular targets amenable to therapeutic intervention as well as lead molecules with health-promoting potential (Kritzer et al. 2009; Su et al. 2010; Tardiff et al. 2012; Menezes et al. 2015). BacHBerry aimed at identifying phenolic bioactives from harnessing the diversity of phenolics in selected berry germplasm from cultivated, wild and underutilized species of berries. The yeast-based screening platform for bioactivity identification comprised a set of genetically-modified $S$. cerevisiae strains expressing Green Fluorescent Protein (GFP) fused with A $\beta 42$ (Bharadwaj et al. 2010), aSyn (Outeiro and Lindquist 2003), HTT (Krobitsch and Lindquist 2000) and FUS (Ju et al. 2011) under the control of a galactose-inducible promoter (Fig. 3a). Upon activation of expression, these proteins start forming aggregates, which consequently has a negative impact on cellular growth and results in lower GFP fluorescence levels. In case an extract possesses bioactivity against one of the diseases, addition of the extract to the activated yeast cells reduces growth inhibition in the corresponding model. These yeast strains accelerated the identification of phenolic compounds with health-promoting attributes among the most chemically diverse samples identified by the metabolomic analysis (Table 2). For information on the procedures used during the screening see Supplementary Materials S1.

An illustrative example of how bioactivities for AD were identified is given in Fig. 4a. Upon shift of cells to galactose medium to induce expression of GFP$\mathrm{A} \beta 42$, yeast growth was impaired in comparison to the control strain indicating GFP-A $\beta 42$ proteotoxicity. The treatment with polyphenol-enriched extracts of Lycium chinense significantly recovered growth of these cells revealing its protective role via modulation of $\mathrm{A} \beta 42$ toxicity. The bioactivities for PD, HD and ALS were screened using a similar approach, in cells expressing the respective disease proteins.

In addition, we have also used a yeast-based model for inflammatory signaling that is based on a Crz1 reporter-strain (Prescott et al. 2012; Garcia et al. 2016; Fig. 3b). Crz1 is a yeast orthologue of NFAT, which is an important modulator of inflammation in humans that is known to be involved in development of multiple disorders, such as the inflammatory bowel disease or the rheumatoid arthritis (Pan et al. 2013). Both Crz1 and NFAT are known to be activated by a serine/threonine protein phosphatase calcineurin $(\mathrm{CaN})$ in a $\mathrm{Ca}^{2+}$-dependent manner (Rusnak and Mertz 2000; Bodvard et al. 2013). The utilized reporter strain encodes the $\beta$-galactosidase gene (lacZ) under the control of a promoter bearing Crz1-binding sites, the Calcineurin-Dependent Responsive Element (CDRE), allowing the assessment of Crz1 activation through the measurement of $\beta$-galactosidase activity using chromogenic substrates (Garcia et al. 2016). Given the evolutionary conservation of NFAT and Crz1 activation mechanisms, in combination with the conserved activity of the immunosuppressant FK506 both in yeast and in humans, the yeast $\mathrm{Ca}^{2+} / \mathrm{CaN} / \mathrm{Crz} 1$ reporter assay represents a reliable tool to identify 


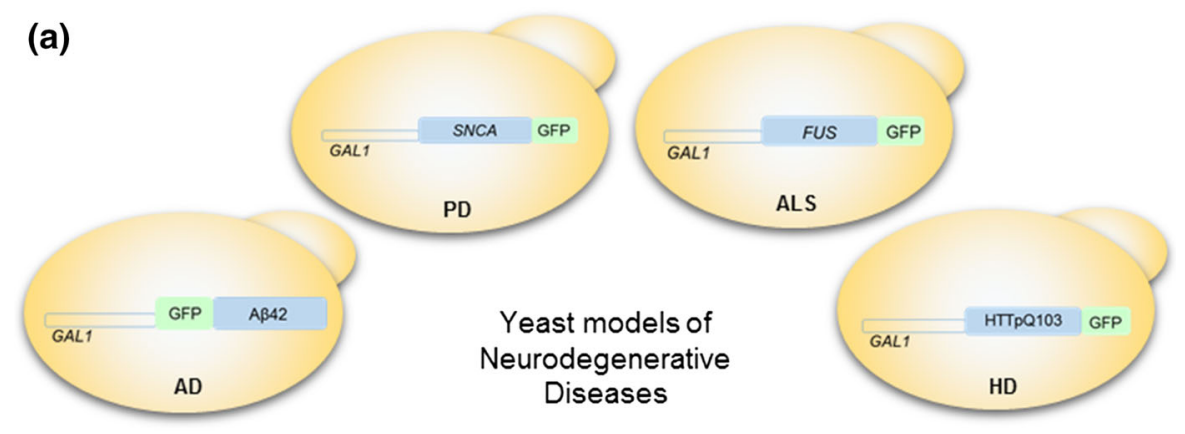

(b)

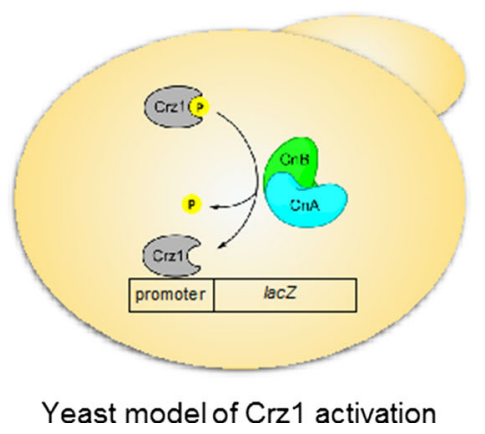

Fig. 3 Schematic representation of yeast models. a Yeast models of neurodegenerative diseases. Humanized yeast strains encoding chimeric fusions of disease genes with GFP (green fluorescent protein) under the control of a galactose-inducible (GAL1) promoter. Expression of A $\beta 42$, $\alpha$ Syn (SNCA gene), FUS and HTTpQ103 leads to the formation of toxic aggregates, recapitulating the pathological processes of Alzheimer's disease (AD), Parkinson's disease (PD), Amyotrophic lateral sclerosis

bioactives with potential to attenuate NFAT-mediated inflammatory responses. The potential of Lycium chinense polyphenol-enriched extracts to attenuate inflammation is shown in Fig. 4b, exemplifying the approach used in BacHBerry to filter for potential antiinflammatory activities. The activation of Crz1 by $\mathrm{MnCl}_{2}$, mimicking NFAT activation, led to high $\beta$ galactosidase activity and cell treatment with FK506 and Lycium chinense extract strongly reduced the $\beta$ galactosidase levels revealing its ability to modulate Crz1, and potentially NFAT, activation.

These examples illustrate the strategy used in the BacHBerry project to search for bioactive compounds interfering with pathological processes of NDs and inflammation. The yeast-based screening platform also included strains allowing the identification of metabolites with potential application for type II diabetes, hematological diseases and cancer (unpublished results).
(ALS), and Huntington's disease (HD), respectively. b Yeast model of Crz1 (NFAT orthologue) activation. Crz1 is activated by the serine/threonine protein phosphatase calcineurin (composed by $\mathrm{CnA}$ and $\mathrm{CnB}$ subunits). The reporter strain encodes the lacZ gene under the control of a promoter containing Crz1binding sites, allowing the assessment of Crz1 activation through the measurement of $\beta$-galactosidase activity

Bioassay-guided fractionation compound discovery and candidate compound validation

This method of discovering new bioactive natural products ultimately depends on the availability of biological material and preparative- or semi-preparative-scale analytical methods with the capability of resolving mixtures of different classes of compounds typically present in berry extracts (Pauli et al. 2012). Semi-preparative chromatography was used to fractionate selected berry extracts with potential bioactive properties using a hybrid approach of bioassay-guided fractionation procedure (Yang et al. 2001; Weller 2012; Tayarani-Najaran et al. 2013) and screening of pure compounds (Watts et al. 2010). Bioassay-guided fractionation typically involves the following steps: assessment of bioactivity, extraction of the biological material with different solvents and testing of bioactivity. Once bioactivity of an extract has been 
(a)

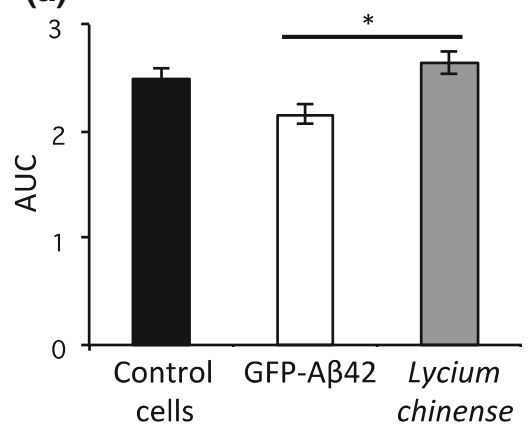

Fig. 4 Identification of bioactivities in the berry germplasm. a Strategy used for the discovery of bioactive compounds for Alzheimer's disease. BY4741_erg6 recombinant yeast cells expressing GFP-A $\beta 42$ were grown in SD galactose medium and cell viability was assessed by growth curves for $24 \mathrm{~h}$ in cells challenged or unchallenged with Lycium chinense polyphenol extracts. Cells containing the empty vector were used as controls. The areas under the curve (AUC) were integrated using the Origin software (OriginLab, Northampton, MA). Values represent the mean \pm standard error of mean (SEM) of at least

validated, the extract gets subjected to an iterative process of sub-fractionation/bioactivity testing until pure bioactive natural products are obtained for structural characterization. This approach benefits from exclusion of extracts that do not have bioactivity. However, this procedure requires extensive use of biological material and expensive materials and may result in the isolation of already-known natural products (Duarte et al. 2012). Another disadvantage is that the approach is based on the assumption that bioactivity is conferred by a pure compound, although this method can also be used for identification of bioactivities conferred by a cumulative interactions of several polyphenols. Alternatively, pure-compound screening relies on an initial isolation and structural elucidation of the individual compounds present in the biological extract followed by bioassay screening. This strategy allows the researcher to focus solely on novel compounds, without re-discovering compounds with well-annotated bioactivity. However, it may also lead to the identification of novel compounds with no bioactivity (Duarte et al. 2012). The second method (b)

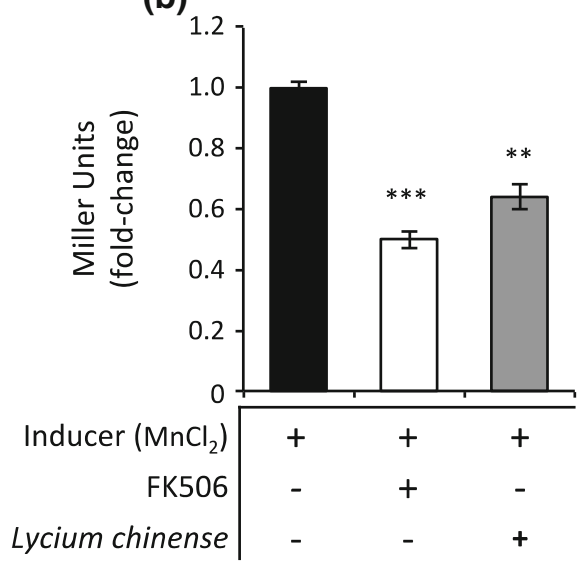

three biological replicates. b Strategy used for the discovery of bioactive compounds with anti-inflammatory potential. BY4741 yeast cells encoding $\mathrm{CDRE}_{\text {promoter- }}-\mathrm{ac} Z$ were treated or not with Lycium chinense polyphenol extracts and induced with $1.8 \mathrm{mM}$ $\mathrm{MnCl}_{2}$. Crz1 activation was assessed by monitoring $\beta$-galactosidase activity using ortho-nitrophenyl- $\beta$-galactoside (ONPG). The immunosuppressant FK506, a well-known inhibitor of calcineurin (Dumont 2000) was used as a positive control. The values represent the mean \pm SEM of at least three biological replicates, ${ }^{*} p<0.05$, ** $p<0.01$, *** $p<0.001$

misses any synergistic interactions affecting bioactivities of berry phenolics.

The limited amount of biological material available restricted the number of iterations of fractionation and re-testing of fraction bioactivity possible for typical bioassay-guided fractionation approaches. As described previously, berry extracts typically comprise a relatively diverse and large pool of metabolites that surpass by far the throughput capability of the bioactivity assays used in this study. To overcome these challenges, a hybrid approach was adopted which consisted of several steps: (1) assessment of potential bioactivity present in extracts (as described above), (2) fractionation of bioactive extracts (see Supplementary Materials S1), (3) assessment of potential bioactivity present in fractions, (4) massspectrometry-based chemical characterization of bioactive fractions, (5) bioactivity testing of pure candidate compounds (Fig. 5). Although this approach shared some of the limitations of the other approaches, it did allow for the exclusion of nonbioactive biological extracts or fractions and focused on the identification novel compounds with potential 
Fig. 5 Yeast as a discovery platform of therapeutic compounds. Iterative bioguided fractionation of complex mixtures allows the discovery of small molecules improving cell growth, by rescue of protein aggregates toxicity, or inhibiting inflammatory processes. High-throughput formats aligned with chemical identification by LC-MS approaches accelerate the identification of candidate compounds

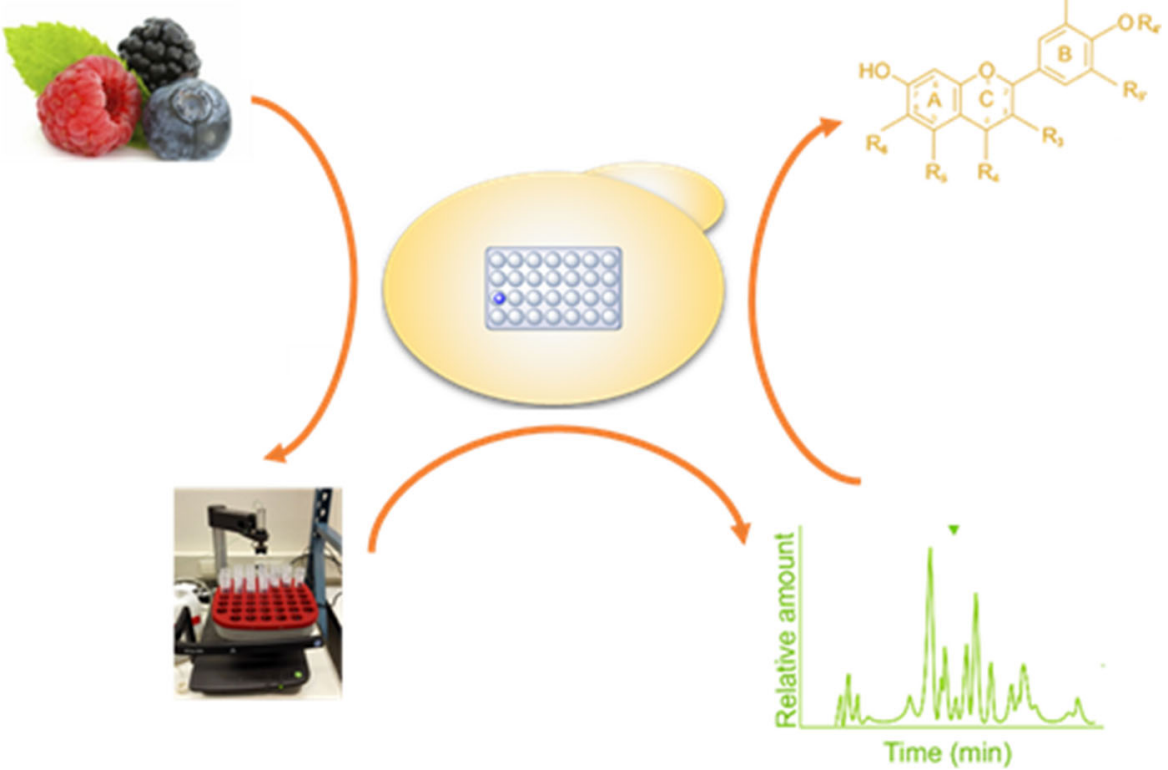

pathways corresponding to the newly discovered bioactives, in order to establish production of these compounds in bacterial hosts. In plants, the biosynthesis of polyphenols occurs via the phenylpropanoid pathway, where the aromatic amino acid L-phenylalanine serves as a precursor. Depending on the enzyme combination, several compounds can be produced in bacteria, including cinnamic acid derivatives, lignin subunits, lignans, phenylpropenes and coumarins, all sharing a basic $\mathrm{C}_{6}-\mathrm{C}_{3}$ skeleton (Vogt 2010). Furthermore, the phenylpropanoid backbone can be extended with up to three acetyl-units derived from malonylCoA by chalcone synthase (CHS) or stilbene synthase (STS), polyketide synthases which generate various polyketides, such as flavonoids and stilbenes. The core pathways of phenolic biosynthesis are very well understood, with common enzymes, such as, phenylalanine ammonia lyase (PAL), cinnamate 4-hydroxylase $(\mathrm{C} 4 \mathrm{H}), p$-coumaroyl CoA ligase (4CL), hydroxycinnamoyl-CoA shikimate/quinate hydroxycinnamoyltransferase (HCT), p-coumaroyl shikimate/quinate $\quad 3^{\prime}$-hydroxylase $\left(\mathrm{C}^{\prime} \mathrm{H}\right), \quad$ stilbene synthase and chalcone synthase, found in many plant species (Fig. 6).

Phenolic chemodiversity in berries, as in other plants, derives from a variety of decorations to the backbone of phenolic structures that include hydroxylations, $O$-methylations, prenylations, aryl migrations, glycosylations, acylations and polymerizations.
The next step within the BacHBerry pipeline involved the identification of the biosynthetic genes and

\section{Identification of metabolic pathways and regulators involved in phenolic production}




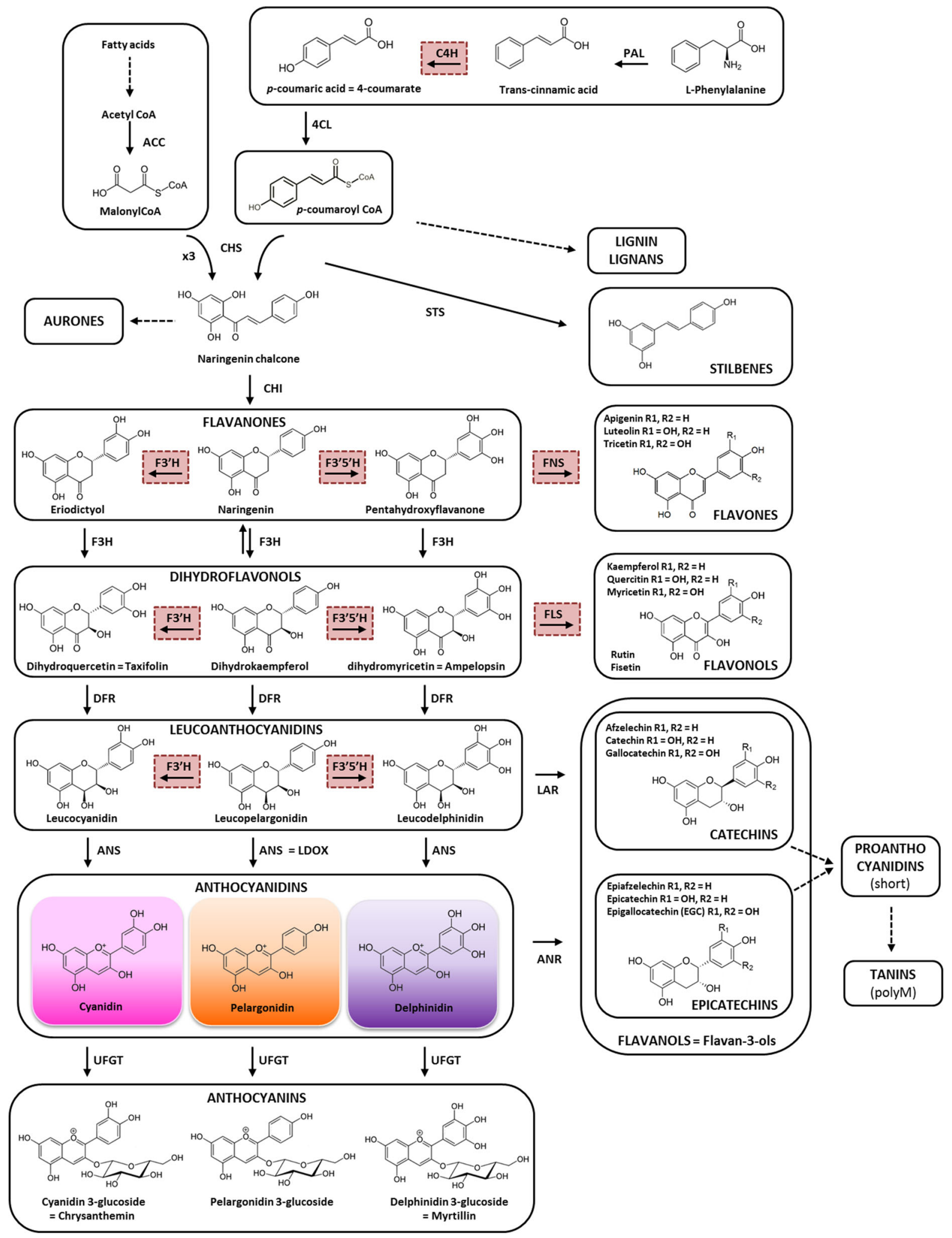


4Fig. 6 Polyphenol biosynthetic pathway in plants (modified from Falcone Ferreyra et al. 2012). $P A L$ phenylalanine ammonia-lyase, $C 4 H$ cinnamate 4-hydroxylase, $4 C L$ 4-coumaroyl-CoA ligase, $\mathrm{CHS}$ chalcone synthase, $\mathrm{CHI}$ chalcone isomerase, $F 3 H$ flavanone 3-hydroxylase, $F 3^{\prime} 5^{\prime} H$ flavonoid $3^{\prime}-5^{\prime}$ hydroxylase, DFR dihydroflavonol 4-reductase, ANS anthocyanidin synthase, ANR anthocyanidin reductase, UFGT flavonoid 3-O-glucosyltransferase, $L A R$ leucoanthocyanidin reductase, FNS flavone synthase, FLS flavonol synthase, STS stilbene synthase. P450 enzymes are highlighted in red

For example, anthocyanins are glycosylated on 3- $\mathrm{OH}$ positions of the C-ring (Fig. 1) and many harbor additional glycosyl groups (for example $5^{\prime}$ - $O$-glycosylation and $3^{\prime}-O$-glycosylation), methoxy groups (petunidin and malvinidin), or hydroxyl groups [which differentiate pelargonidin, cyanidin and delphinidin (Fig. 6; Table 1)]. These decorations may affect the bioavailability, bioactivity and stability of polyphenolic compounds. It is therefore essential to develop a greater knowledge of the decorating enzymes specific to berry bioactives, such as those differentially decorating anthocyanins, flavonols, stilbenes, or condensed tannins. Many genes encoding enzymes involved in flavonol and anthocyanin decoration have already been characterized not only in planta, but also heterologously in microorganisms, such as Escherichia coli and S. cerevisiae (Tohge et al. 2005; Luo et al. 2007; Pandey et al. 2016; Wang et al. 2016a).

Berry bioactives identified within the project had special decorations, and those where the genes encoding the decorating activities had not been previously identified, required further research. In a recent work, the transcriptome of the commercial blueberry (Vaccinium corymbosum) was studied using RNA sequencing technology (RNA-seq) in order to discover putative genes related to antioxidant production using next-generation sequencing (NGS; Li et al. 2012). Similarly, we aimed to identify decorating enzymes and regulators of the biosynthetic pathways of berry bioactives by obtaining transcriptome sequences of target species at different stages of fruit development. This information was used to construct a database of transcriptome sequences for a broad range of berry species, especially those identified as target species containing the promising bioactive molecules. In addition, the overall production of polyphenols is regulated by a range of transcription factors and these were also investigated within the BacHBerry project, with the objective of identifying markers for breeding enhanced levels of phenolic bioactives using the diverse germplasm in selected berry species.

Firstly, a library of reference sequences consisting of plant gene sequences encoding enzymes of the core phenolic biosynthetic pathways, which was subsequently used for BLAST searches of berry transcriptomes, was compiled. This library included wellknown and experimentally validated anthocyanin, flavonol, stilbene and catechin biosynthetic enzymes from a wide range of plant species. Next, 13 germplasms spanning eight genera, seven families and seven orders were selected for RNA-seq of berry transcriptomes. Sequencing of selected berries at different stages of maturation was also conducted. The data have provided significant resources not only for understanding phenolic biosynthesis in berries, but also for investigating commonalities in genes expressed in berries (Table 3).

Multi-layer comparisons of the metabolomics and transcriptomics data of developmental stages, and targeted, as well as, non-targeted searches of candidate transcripts were performed to elucidate genes involved in specific phenolics biosynthetic pathways. Analyses of expression of these genes from different developmental stages of selected berry species were performed with the expVIP tool (Borrill et al. 2016).

These data were used to search for genes encoding putative decorating enzymes based on the list of molecules of potential interest as bioactives and having special decorations in target berry species. Candidates were identified mostly by searching for homologues of well-characterized plant genes. This was carried out with the help of MassBlast, a software developed within the BacHBerry project (https:// github.com/averissimo/mass-blast; Veríssimo et al. 2017). We annotated all major enzyme families, including cytochromes P450 (CYP), 2-oxoglutaratedependent dioxygenases (2-OGDs), UDP-glycosyltransferases (UGTs), BAHD acyltransferases, and $O$ methyltransferases (OMTs), in all 13 selected berry germplasms. Average counts per transcriptome are presented in Table 4 . We also detected candidate transcript(s) for $60-80 \%$ of the known biochemical steps of the phenylpropanoid pathway, representing between 109 and 180 candidates per transcriptome. Approximately $40 \%$ of these candidates identified from the transcriptomes were full-length sequences. 
Table 3 Transcriptome resources for berries developed on BacHBerry

\begin{tabular}{|c|c|c|}
\hline Species & Plant material & Total number of raw reads \\
\hline Aristotelia $s p$. & Ripe berries & $397,707,372$ \\
\hline Berberis sp. & Ripe berries & $444,362,698$ \\
\hline Corema sp. & Leaf & $353,604,932$ \\
\hline Lonicera sp. & Ripe berries & $397,214,254$ \\
\hline Ribes sp. 1 & Ripe berries & $336,479,242$ \\
\hline Ribes sp.2 & Ripe berries & $393,665,630$ \\
\hline Rubus sp. 1 & Berries (3 stages) & $1,040,224,680$ \\
\hline Rubus sp.2 & Berries (3 stages) & $1,064,858,518$ \\
\hline Rubus sp.3 & Ripe berries & $505,754,030$ \\
\hline Rubus sp.4 & Ripe berries & $390,608,452$ \\
\hline Ugni sp. & Ripe berries & $405,024,920$ \\
\hline Vaccinium sp.1 & Ripe berries & $373,159,882$ \\
\hline Vaccinium sp.2 & Ripe berries & $375,778,718$ \\
\hline
\end{tabular}

Table 4 Statistics on five major enzyme families representing conserved biochemical steps of the phenylpropanoid pathway

\begin{tabular}{lc}
\hline Enzyme families & Candidates per transcriptome (min-max) \\
\hline Conserved phenylpropanoid pathway enzymes & $109-180$ \\
CYPs & $100-177$ \\
UGTs & $70-145$ \\
2-OGDs & $32-88$ \\
$O$-methyltransferases & $8-51$ \\
Acyltransferases & $23-36$ \\
\hline
\end{tabular}

Candidates displayed more than $70 \%$ sequence coverage to reference query sequences. This work led to the creation of a large dataset of candidates used for pathway elucidation and reconstitution in bacterial hosts

Furthermore, biochemical activities of some of enzymes encoded by the candidate genes were tested.

We also looked for transcripts encoding regulators of phenolic biosynthesis. Since the R2R3-MYB gene family operates extensively in controlling phenylpropanoid metabolism in plants (Stracke et al. 2001; Hartmann et al. 2005), initial searches were focused on identifying MYB-genes that were strongly expressed in berries. However, members of the bHLH and bZIP families of transcription factors were also identified in the berry RNA-seq databases since they may also play roles in regulating accumulation of phenolic bioactives (Hartmann et al. 2005). These data could be particularly useful in breeding programs for selection of plants with improved bioactive contents. The presence of genes encoding putative transporters of phenolic compounds was also examined. Excretion of target compounds produced by bacterial hosts into the culture medium via such transporters would simplify extraction procedures, and may also be necessary in case of any toxic effects of these molecules in bacteria. One transporter is currently being characterized functionally.

\section{Gram-positive bacteria as cell factories for the efficient production of berry high-value phenolics}

While the search for novel bioactives was under way, a set of well-characterized biosynthetic pathway was used for establishing a platform for production of phenolic compounds in Gram-positive bacteria. Two major criteria were used for the selection of host organisms for this task: (1) they had to be robust, wellstudied organisms with a long-standing history of 
industrial use and (2) the bacteria must have a generally regarded as safe (GRAS) status and/or be recognized as food-grade. Two industrially-important bacteria, namely Lactococcus lactis and Corynebacterium glutamicum, appeared as attractive candidates for the sustainable production of value-added food and pharmaceutical compounds (Lahtinen et al. 2011; Jojima et al. 2013). Both L. lactis and C. glutamicum are used worldwide in numerous industrial processes, which consequently led to significant amount of research work dedicated towards improving our knowledge of their physiology and genetics. These efforts have resulted in development of large toolboxes for (heterologous) gene expression and introduction of genetic modifications in these bacteria (Burkovski 2008; Pontes et al. 2011; Gaspar et al. 2013). Furthermore, high-throughput methodologies for transcriptome, proteome and metabolome analysis, and genome-scale metabolic models are available for these species (Krömer et al. 2004; Oliveira et al. 2005; Shinfuku et al. 2009; Gaspar et al. 2013). Combined with their general robustness, ease of handling and the GRAS status, both L. lactis and C. glutamicum have a strong potential for becoming platform organisms for production of polyphenolic compounds.

It should also be noted that during the earlier stages of the project, E. coli was also exploited as a platform for reconstruction of polyphenol biosynthetic pathways. E. coli has a long history of use in research on microbial-based production of various value-added chemicals. Moreover, it is one of the best studied organisms, easy to handle, and a much larger set of genetic tools is available for this organism compared to other bacteria (Yu et al. 2011; Dobson et al. 2012; Chen et al. 2013). Furthermore, E. coli has been previously used for heterologous production of polyphenolic compounds (Pandey et al. 2016; Wang et al. 2016a).

\section{Lactococcus lactis}

Lactococcus lactis is a Gram-positive bacterium from the order of Lactobacillales that has been widely used in the food industry for the manufacturing of cheese, buttermilk and other dairy products. It is a low $\mathrm{G}+\mathrm{C}$ aerotolerant bacterium with a relatively small genome and simple sugar metabolism: utilization occurs via glycolysis (Embden-Meyerhof-Parnas pathway) with lactic acid being the major end-product (Gaspar et al.
2013). Its ability to ferment various carbohydrates, such as hexoses, pentoses and, in some strains, even complex carbohydrates of plant origin, as well as its high alcohol, acid and stress tolerance makes this bacterium suitable for the harsh environments commonly found under industrial production conditions. Lactococcus lactis strains have been engineered to produce multiple commodity chemicals (e.g. 2,3butanediol and butanol), as well as food additives, including sweeteners (e.g. mannitol and xylitol), flavorings (e.g. acetaldehyde and diacetyl), and vitamins. Furthermore, production of plant secondary metabolites, namely isoprenoids and stilbenes, has been demonstrated in this organism (Neves et al. 2005; Katz et al. 2011; Gaspar et al. 2013; Song et al. 2014). In addition, L. lactis is well known as an excellent host for the expression of both prokaryotic and eukaryotic proteins, and has been consequently used for development of live vaccine (Kunji et al. 2005; Hernández et al. 2007; Frelet-Barrand et al. 2010; Pontes et al. 2011).

As depicted in Fig. 6, in plants the biosynthesis of the core intermediate of the phenylpropanoid pathway, $p$-coumaric acid, begins from L-phenylalanine and occurs in two steps: a deamination reaction catalyzed by phenylalanine ammonia-lyase (PAL) and a hydroxylation reaction catalyzed by cinnamate 4-hydroxylase $(\mathrm{C} 4 \mathrm{H})$. The latter enzyme belongs to the CYP family that is often challenging to express in active form in prokaryotic systems (Dvora and Koffas 2013). However, in some bacteria and fungi there exists an alternative pathway where L-tyrosine is converted directly into $p$-coumaric acid via the action of tyrosine ammonia-lyase (TAL), hence bypassing the $\mathrm{C} 4 \mathrm{H}$ step (Fig. 6). As a first step towards establishing a platform strain for the production of polyphenols in L. lactis, in collaboration with Nielsen and coworkers, we identified several novel TAL enzymes that specifically produce high levels of $p$-coumaric acid from $\mathrm{L}$ tyrosine (Jendresen et al. 2015).

To test the potential of L. lactis to produce more complex phenolic compounds, we introduced a pathway for the biosynthesis of resveratrol. The introduced pathway consisted of three enzymes: TAL, 4CL (4coumaroyl-CoA ligase, which converts $p$-coumaric acid to $p$-coumaroyl-CoA), and STS (stilbene synthase, which catalyzes the condensation of $p$-coumaroyl-CoA and three molecules of malonyl-CoA to yield trans-resveratrol, Fig. 6). The corresponding genes 
were placed on the high copy number vector pNZ8048 under the control of the nisin-inducible promoter $\mathrm{P}_{\text {nisA }}$. This promoter is a part of a tightly-controlled inducible gene expression system which is based on the auto-regulatory mechanism controlling the production of the bacteriocin nisin (Kuipers et al. 1993, 1998). The producer L. lactis strain contains a chromosomally-integrated two-component system NisRK allowing induction of its target promoters by addition of sub-inhibitory concentrations of the bacteriocin nisin to the exponentially-growing cell culture (Linares et al. 2010). Upon cultivation of the producer strain in a chemically defined medium, production of trans-resveratrol was detected in the order of several $\mathrm{mg} \mathrm{l}^{-1}$. When TAL was replaced with a promiscuous PAL/TAL enzyme (MacDonald and D'Cunha 2007), additional production of small amounts of pinosylvin (trans-3,5-dihydroxystilbene) from L-phenylalanine was observed. Furthermore, production of methylated variants of trans-resveratrol was also achieved (unpublished results). Obtained titers for trans-resveratrol are comparable to those obtained in an early work on trans-resveratrol production in both E. coli and yeast (Beekwilder et al. 2006; Choi et al. 2011). Current research efforts focus on improving the production efficiency through metabolic engineering of the central carbon metabolism for increased precursor supply.

Anthocyanins (glycosylated anthocyanidins) are another group of phenolic phytochemicals. In addition to their antioxidant properties, anthocyanins are valuable for their colors, which range from orange to blue (Falcone Ferreyra et al. 2012). They are of particular interest for the food industry as natural alternatives to replace synthetic dyes. Anthocyanins are synthesized from flavanones by flavanone 3-hydroxylase $(\mathrm{F} 3 \mathrm{H})$, which adds a hydroxyl group to the C3 position. Resulting dihydroflavonols are further processed by dihydroflavonol 4-reductase (DFR), which catalyzes reduction of the carbonyl group of the $\mathrm{C} 4$ position to form leucoanthocyanidins, which in turn are converted to anthocyanidins via the action of anthocyanidin synthase (ANS). Anthocyanidins are further stabilized through a C3-glycosylation reaction usually catalyzed by UDP-glucose:flavonoid 3-Oglucosyltransferase (UFGT) to form the anthocyanidin 3-O-glucosides (Falcone Ferreyra et al. 2012; Fig. 6).

In order to reconstruct the anthocyanin production pathway in L. lactis, native genes and genes codonoptimized for L. lactis from various plant species coding for F3H, DFR, ANS and UFGT were placed under control of the nisin-inducible promoter $\mathrm{P}_{\text {nisA }}$ on a high copy-number vector or were integrated into the chromosome of L. lactis. Each protein was tagged with an $\mathrm{N}$ - or $\mathrm{C}$ - terminal strep-tag, and Western blots were performed to detect the heterologous proteins in cellfree extract of $L$. lactis. Activity of each enzyme was probed in vitro and in vivo and the reaction products were analyzed using high performance liquid chromatography (HPLC). Production of various types of cyanidin-derived anthocyanidins and anthocyanins by the engineered L. lactis strains was detected, further demonstrating the potential of this lactic acid bacterium as a production host for plant-derived bioactive compounds for food applications (unpublished results). To our knowledge, this is the first report of using lactic acid bacteria for the production of complex phenolic compounds.

Malonyl-CoA is a rate-limiting precursor in the biosynthesis of various phenolic compounds in bacteria (Xu et al. 2011; Bhan et al. 2013). Under physiological conditions, L. lactis cells use malonyl$\mathrm{CoA}$ as a substrate in the chain elongation step of the fatty acid biosynthesis. FapR of Bacillus subtilis is a transcriptional repressor that directly senses the intracellular pool of malonyl-CoA. A metabolic biosensor based on the interaction of malonyl-CoA with FapR was previously designed in Escherichia coli (Xu et al. 2013). As a part of the efforts to enhance polyphenol production, a transcriptional biosensor for malonylCoA was constructed for the use in C. glutamicum but also proved to be fully functional in L. lactis and could therefore be used for screening of variants with an enhanced malonyl-CoA pool (unpublished results).

Among other activities that were pursued in the early stages of the project, we also tried to express plant CYPs in L. lactis, as many steps of the polyphenol biosynthetic pathway are catalyzed by these heme-bound monooxygenases. Functional expression of genes encoding membrane-anchored CYPs in prokaryotes is known to be notoriously difficult, and is limited by problems associated with the differences in intracellular localization (natively to the plant endoplasmatic reticulum), membrane composition, protein folding, and cofactor utilization. The latter element became especially crucial when attempting to express these proteins in L. lactis, because this, normally anaerobic, bacterium does not have the ability to synthetize heme, despite 
undergoing a metabolic shift to respiration when supplemented with an exogenous source of heme and being capable of regulating heme homeostasis (Pedersen et al. 2012).

We focused our efforts on CYP79A1 from Sorghum bicolor as it is a well-characterized CYP involved in dhurrin biosynthesis (Bak et al. 2000), and has been previously successfully produced in E. coli (VazquezAlbacete et al. 2016). As a testbed for the expression of this CYP we have used the Waldo platform (Waldo et al. 1999) that was previously instrumental in producing functional CYP79A1 in E. coli. Briefly, the platform is based on a fusion of the membrane protein with a GFP folding reporter at the $\mathrm{C}$ terminus: the fused GFP is designed to act as a fluorescent reporter only when the membrane protein is properly folded and its $\mathrm{C}$ terminus is oriented towards the cytoplasm.

We detected CYP79A1-GFP by both fluorescence measurements of the L. lactis culture and in-fluorescence protein gels, but were not able to detect activity of the CYP in our in vitro tests. Purified membrane protein fractions from cell cultures grown both aerobically (with hemin supplementation) and anaerobically (with addition of hemin after purification of membrane proteins) did not show any product formation in our TLC-based assay (Blomstedt et al. 2012). These results suggested that CYP79A1 was probably misfolded in L. lactis, although we did not perform any follow-up experiments to confirm this hypothesis. The topic was not pursued any further because any CYP active in the biosynthetic pathways for the selected model compounds could be by-passed (for example the $\mathrm{C} 4 \mathrm{H}$-catalyzed step could be by-passed by using TAL instead of PAL).

\section{Corynebacterium glutamicum}

Corynebacterium glutamicum is an important organism in industrial biotechnology for the microbial production of bulk chemicals, in particular of amino acids (Becker and Wittmann 2012). C. glutamicum is a very robust microorganism, which shows high resistance to the presence of small aromatic compounds (Liu et al. 2013), which renders $C$. glutamicum a very promising host for the production of pharmacologically interesting plant-derived polyphenols (Marienhagen and Bott 2013). Until recently, the activity of a complex catabolic network for aromatic compounds meant that $C$. glutamicum was not used for the production of aromatic compounds (except for aromatic amino acids; Shen et al. 2012) due to rapid degradation of the products of interest. However, $C$. glutamicum can be easily modified genetically and a toolbox for the high-level expression of heterologous genes originating from other organisms, has become available, and is based on a set of strong and inducible promoters (Eggeling and Bott 2005; Pátek and Nešvera 2013; Kortmann et al. 2015).

Surprisingly, in initial experiments, it turned out that $C$. glutamicum is able to grow on naturally occurring phenylpropanoids such as $p$-coumaric acid, ferulic acid, caffeic acid and 3-(4-hydroxyphenyl)propionic acid as sole carbon and energy sources. The maximum growth rates on the selected phenylpropanoids ranged from 0.15 to $0.23 \mathrm{~h}^{-1}$ corresponding to doubling times of 3-4.5 h (Kallscheuer et al. 2016a). We suspected that the activity of this pathway would come into conflict with any attempt to produce polyphenols as both pathways compete for phenylpropanoids. Unfortunately, the underlying metabolic pathway responsible for the degradation of phenylpropanoids in C. glutamicum was unknown at the start of the BacHBerry project. Global gene expression analyses were conducted, which led to the identification of a gene cluster consisting of seven genes (designated $p h d T, p h d A, p h d R, p h d B$, phdC, phdD and $p h d E)$. In response to feeding of the above-mentioned phenylpropanoids these genes were upregulated up to 100-fold (Kallscheuer et al. 2016a). In subsequent studies, it turned out that the genes in this cluster code for the enzymes of the phenylpropanoid catabolic pathway in C. glutamicum. The gene $p h d T$ codes for a phenylpropanoid transporter protein, whereas $p h d A$ and the predicted operon $p h d B C D E$ code for enzymes involved in the degradation of phenylpropanoids. The phd gene cluster is transcriptionally controlled by a MarR-type repressor encoded by phdR. Cultivation experiments conducted with $C$. glutamicum strains carrying single-gene deletions showed that loss of phdA, phdB, phdC or phdE abolished growth of $C$. glutamicum with all tested phenylpropanoid substrates. The intracellular accumulation of pathway intermediates determined via LC-ESI-MS/MS in single-gene deletion mutants showed that the phd gene cluster encodes for enzymes involved in a CoAdependent, $\beta$-oxidative deacetylation pathway, which is essential for the utilization of phenylpropanoids in 
C. glutamicum. The identified pathway thereby represents a peripheral catabolic route responsible for chain shortening of phenylpropanoids to benzoic acids and acetyl-CoA (Fig. 7). C. glutamicum converts the resulting intermediates benzoate, 4-hydroxybenzoate and protocatechuate to succinyl-CoA and acetyl-CoA by central degradation pathways for aromatic compounds (especially the well-characterized $\beta$-ketoadipate pathway), and catabolites are ultimately channeled into the tricarboxylic acid cycle (Shen and Liu 2005; Fig. 7). Unexpectedly, C. glutamicum failed to grow on the phenylpropanoid, cinnamic acid, which lacks ring para-hydroxylation. Astonishingly, a $C$. glutamicum strain with a single deletion of the gene phdR coding for the identified transcriptional repressor was able to grow with cinnamic acid as sole carbon and energy source (Kallscheuer et al. 2016a). This indicated that PhdR has a pronounced specificity for hydroxylated phenylpropanoids, especially when hydroxylated in para-position of the aromatic ring. In the course of our studies, cinnamic acid was the only phenylpropanoid tested lacking this hydroxylation, so the complete specificity of this catabolic pathway of phenolics in C. glutamicum remains unknown.

During construction of the C. glutamicum platform strain for plant polyphenol production, four gene clusters, comprising 21 genes, including four genes of the $p h d$ cluster involved in the catabolism of aromatic compounds, were deleted. The resulting platform strain C. glutamicum DelAro ${ }^{4}$ was unable to degrade any phenylpropanoid tested, and also could not utilize simple benzoic acids such as protocatechuate (Kallscheuer et al. 2016c). This strain was used as a chassis for the microbial production of polyphenols, in particular, stilbenes and (2S)-flavanones, in $C$. glutamicum.

Plasmid-borne expression of codon-optimized genes coding for a 4CL from parsley (Petroselinum crispum), a chalcone synthase (CHS) and a chalcone isomerase (CHI) from Petunia $x$ hybrida from the strong T7 promoter in this strain background enabled production of ( $2 S$ )-flavanones (Fig. 7). Maximal titers of $35 \mathrm{mg} \mathrm{l}^{-1}$ naringenin and $37 \mathrm{mg} \mathrm{l}^{-1}$ eriodictyol were obtained from the supplemented phenylpropanoids $p$-coumaric acid and caffeic acid, respectively. Expression of the $4 \mathrm{cl}$ gene in combination with a codon-optimized gene coding for an STS from peanut (Arachis hypogaea) allowed for the production of the stilbenes pinosylvin, trans-resveratrol and piceatannol starting from supplemented phenylpropanoids cinnamic acid, $p$-coumaric acid and caffeic acid, respectively (Kallscheuer et al. 2016c; Fig. 7). Stilbene concentrations of up to $158 \mathrm{mg} \mathrm{l}^{-1}$ could be achieved in defined CGXII medium with $4 \%$ glucose and supplemented with cerulenin, a fatty-acid biosynthesis inhibitor that is known to enhance polyphenol production in bacteria by increasing the intracellular concentrations of malonyl-CoA (Santos et al. 2011; van Summeren-Wesenhagen and Marienhagen 2015; Cao et al. 2016). Engineering amino acid metabolism for an optimal connection to the synthetic plant polyphenol pathways was conducted to enable stilbene production directly from glucose. Indeed, the additional heterologous expression of aroH from $E$. coli (coding for the first enzyme of the shikimate pathway responsible for the synthesis of aromatic amino acids) together with a codon-optimized gene coding for a TAL from Flavobacterium johnsoniae led to the production of up to $60 \mathrm{mg} \mathrm{l}^{-1}$ trans-resveratrol in the absence of supplemented $p$-coumaric acid (Kallscheuer et al. 2016c). Furthermore, C. glutamicum was used to produce trans-resveratrol from a cheaper precursor 4-hydroxybenzoic acid (HBA) through reversal of a microbial $\beta$-oxidative phenylpropanoid degradation pathway (Kallscheuer et al. 2016b). This novel synthetic pathway circumvents any need for the endogenous supply of aromatic amino acids as polyphenol precursors and in C. glutamicum instantly yielded $5 \mathrm{mg}^{-1}$ trans-resveratrol from supplemented HBA without any further optimization. Very recently, we also engineered $C$. glutamicum to produce more complex polyphenols including flavonols (kaempferol and quercetin) and $O$-methylated stilbenes (pinostilbene and pterostilbene). The obtained pterostilbene titer was $42 \mathrm{mg} \mathrm{l}^{-1}$, which is comparable to the titer of $50 \mathrm{mg} \mathrm{l}^{-1}$ obtained in engineered E. coli. In C. glutamicum, for the flavonols kaempferol and quercetin titers of 23 and $10 \mathrm{mg} \mathrm{l}^{-1}$, respectively, were detected. The aforementioned titers were obtained from the phenylpropanoids $p$-coumaric acid and caffeic acid, respectively, as precursors and represent the highest flavonol titers obtained in engineered microorganism until today (Kallscheuer et al. 2017).

The main bottlenecks for the production of polyphenols turned out to be the TAL activity as well as the intracellular availability of malonyl-CoA, 
representing the cosubstrate in the STS- and CHSreactions. On-going efforts to improve polyphenol production in $C$. glutamicum therefore focus mainly on improving the activity of the rate-limiting enzymes and increasing the intracellular malonyl-CoA pool.

\section{Other activities}

As a part of this work, we also aimed at reconstruction of a previously unknown biosynthetic pathway for the flavonol fisetin (3,7,3',4'-tetrahydroxyflavone, Fig. 8). Fisetin is a potent anti-inflammatory, anti-cancer, and anti-oxidant compound (Suh et al. 2008; FunakoshiTago et al. 2011; Jash and Mondal 2014). Moreover, it has been demonstrated to have a neuroprotective effect and also to limit the complications of type I diabetes (Maher 2009; Maher et al. 2011). Based on the molecular structure of this compound, we hypothesized that its biosynthesis should follow the general pathway of flavonol biosynthesis (Figs. 6, 8): a flavanone precursor would be converted into dihydroflavonol by $\mathrm{F} 3 \mathrm{H}$, and then further converted into the corresponding flavonol via the action of flavonol synthase (FLS). In the last step of the biosynthesis, a hydroxyl group would be added at the $\mathrm{C} 3^{\prime}$ position by flavonoid $3^{\prime}$-hydroxylase $\left(\mathrm{F}^{\prime} \mathrm{H}\right)$. This part of the work was carried out in E. coli. Isoliquiritigenin $\left(2^{\prime}, 4^{\prime}, 4-\right.$ trihydroxychalcone) appeared to be the most suitable precursor, and when supplied to an E. coli culture expressing $\mathrm{F} 3 \mathrm{H}$ and FLS from Arabidopsis thaliana, production of garbanzol and resokaempferol was detected. Resokaempferol could be further converted into fisetin by expression of $\mathrm{F}^{\prime} \mathrm{H}$ genes from $A$. thaliana and $P n$. hybrida fused with a cytochrome-

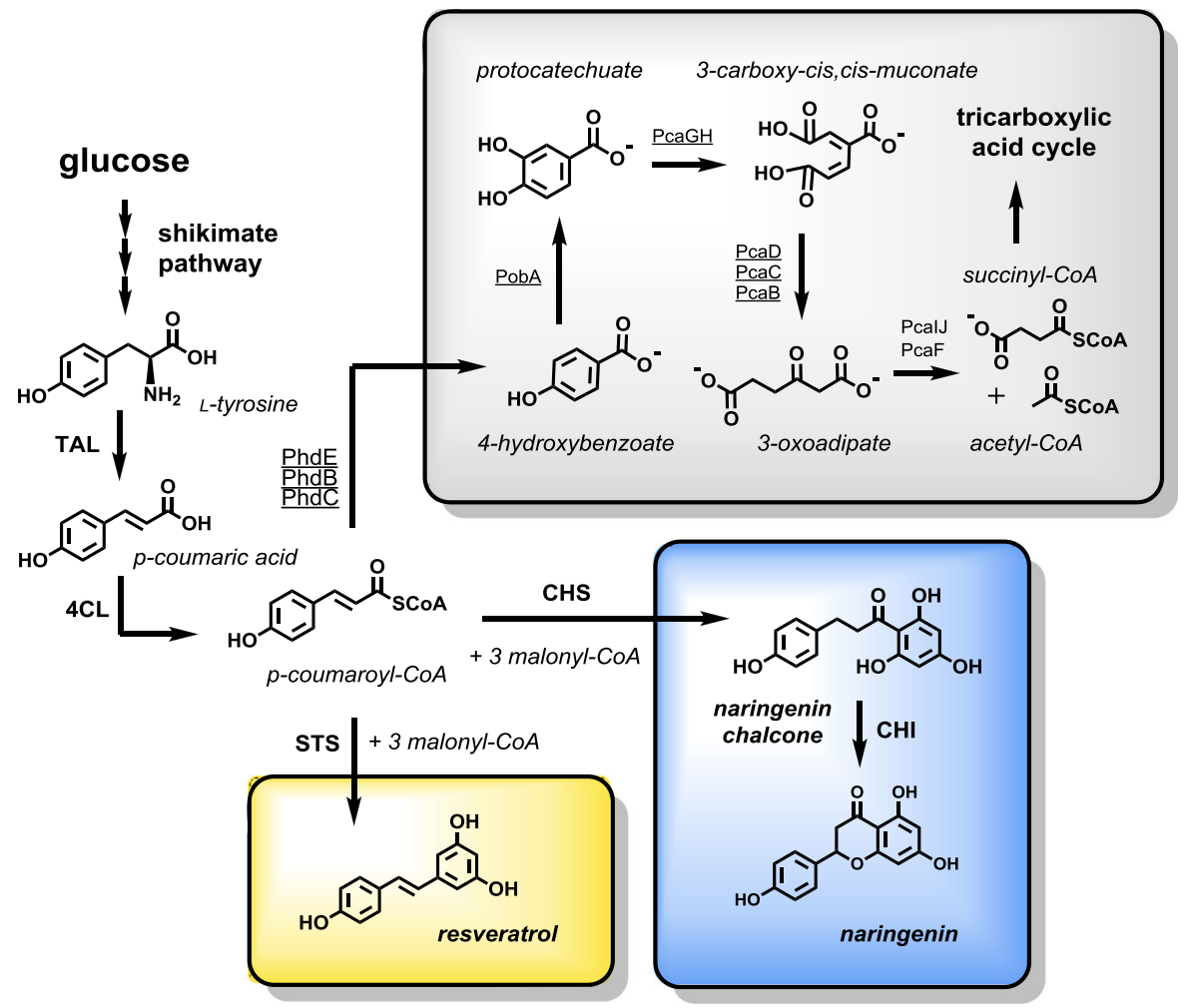

Fig. 7 Overview of the endogenous phenylpropanoid degradation and the engineered pathway for polyphenol synthesis in C. glutamicum. One of the precursors for the production of the stilbene trans-resveratrol (catalyzed by stilbene synthase, STS) or for naringenin chalcone (catalyzed by chalcone synthases, $\mathrm{CHS}$ ) is $p$-coumaroyl-CoA. Naringenin chalcone isomerizes to the $(2 S)$-flavanone naringenin either spontaneously or catalyzed by the activity of chalcone isomerase (CHI). In C. glutamicum, p-coumaric acid can be degraded to 4-hydroxybenzoate by a CoA-dependent, $\beta$-oxidative deacetylation pathway. 4-Hydroxybenzoate is subsequently hydroxylated to protocatechuate, which is catabolized to succinyl-CoA and acetyl-CoA by the $\beta$ ketoadipate pathway. Genes coding for the underlined enzymes were deleted in the course of the construction of the platform strain C. glutamicum DelAro ${ }^{4}$ 
P450 reductase (CPR) from Catharanthus roseus (Stahlhut et al. 2015). The three enzymes were then combined with the isoliquiritigenin biosynthetic pathway, consisting of TAL from Rhodobacter sphaeroides, 4CL from Pr. crispum, CHI from Medicago sativa, and a fusion protein of CHS from Pn. hybrida and CHR from Astragalus mongholicus, to yield $0.3 \mathrm{mg} \mathrm{l}^{-1}$ of fisetin (Stahlhut et al. 2015). This work allowed reconstruction of a complete pathway for the biosynthesis of fisetin from ${ }_{\mathrm{L}}$-tyrosine as the starting precursor using enzymes from various sources, and the identified pathway is currently being transferred to Gram-positive hosts.

\section{Bioinformatics and modelling}

There were multiple activities within BacHBerry, both within the discovery and the microorganism engineering sectors, which required the use of bioinformatics tools. Hence, the mathematical modeling and computational parts of the project aimed at addressing three major problems. The first two were related to gene expression, either for performing functional genomics analysis using the RNA-seq data from berries or to design sequences for heterologous expression of plant genes in microbes. The third problem concerned metabolic network optimization for both a single population and for a community of microbes. We detail below the main results obtained on each of the above activities. Other subjects that were addressed as a part of this project were metabolic communication between the different species in a community (Milreu et al. 2014; Andrade et al. 2016) and modeling dynamic metabolic networks for potential application in strain optimization (Costa et al. 2014; Hartmann et al. 2015a, b, 2016; Costa et al. 2016).

Sequencing data analysis and plant functional genomics

Understanding which genes are differentially expressed during phenolic production by plants requires the capacity to undertake a rapid analysis of RNA-seq data. One of the main problems to be addressed in this case is the search for coding sequences that are homologous to those already present in sequence databases, which involves identification, selection and annotation of the best matches.
Currently, such analyses rely heavily on manual curation as well as on the manual assembly of results obtained using different computational procedures and sequence databases. Automated processes become crucial in the light of the ever-increasing amounts of generated transcriptome data. Furthermore, automation of processes also allows improvement of the quality of the analyses, as the likelihood of human error grows with the amount of manual processing and verifications necessary. Moreover, most of the existing tools are able to annotate the transcriptome but lack the curation functionalities that are as important as the primary homology-based identification of candidate genes.

As mentioned previously we developed a pipeline called MassBlast (Veríssimo et al. 2017), to improve and accelerate the annotation procedure of RNA-seq data. Besides allowing for operators to query multiple databases for performing homology searches, the tool also provides a set of parameters that can be defined in a user-friendly way. MassBlast seeks the best alignment between the sequences in a dataset consisting of contigs obtained from RNA-seq assemblies and a database of known sequences. In the context of BacHBerry and as a general validation of the method, MassBlast was used for the analysis of the blackberry transcriptome, and preliminary results showed a significant improvement in annotation speed $(5 \mathrm{~min}$ vs. at least 26 working days, to annotate all known CYP families in blackberry) with very high accuracies. MassBlast is freely available at https://github. com/averissimo/mass-blast/.

Sequence design for heterologous expression

Synthetic biology aims at constructing microorganisms capable of producing economically-relevant chemical products in a cost-effective way, which involves optimizing the functioning of metabolic pathways and reactions. These pathways and reactions involve proteins that are frequently heterologous. In order to increase the production of metabolites, it is possible to optimize heterologous protein production in the target host. This may be done by altering the codons in the gene that encodes for that protein. Each codon is a sequence of three nucleotides encoding one amino acid that is part of a protein. Since each amino acid can be encoded by several synonymous codons, a 
given protein can be expressed using a large number of different codon combinations.

To address this problem, we worked on improving the machine learning models capable of predicting protein expression levels based on their codon usage frequency (Fernandes et al. 2016) by using Support Vector Regression (SVR) and Partial Least Squares (PLS). SVR yields predictions that surpass those of PLS. It has been shown that it is possible to improve the predictive ability of the models by using two additional input features, namely codon identification number and codon count, besides the already used codon bias and minimum free energy. In addition, applying ensemble averaging to the SVR or the PLS model improved the results even further. Different ensembles and features can be tested with the aim of further improving the prediction models.

Metabolic network optimization

Another goal of the computational activities of BacHBerry was to explore methods for in silico metabolic engineering of phenolic production in $L$. lactis and C. glutamicum. For this we selected four model target compounds: fisetin, trans-resveratrol, pelargonidin and quercetin. Additionally, optimization of malonyl-CoA production was also considered, since this is known to be a major bottleneck in heterologous flavonoid production. The first step involved performing network optimization for a single population, which included prediction of knock-out strategies and determination of minimal sets of genetic interventions that could guarantee an increased production of the target compound production based on stoichiometric models for the two organisms. These predictions were compared with experimental results and model refinement was conducted in cases of discrepancies. For the second step, we considered a use of microbial consortia for production purposes. A method was developed that is being used for modeling the production of the compounds of interest for BacHBerry using L. lactis and C. glutamicum. Other, possibly larger consortia, may be considered in the future.

\section{Single strain-based production}

Recently, several computational methods for in silico metabolic network optimization have emerged and are currently being applied in metabolic engineering (Vera et al. 2010; Zomorrodi et al. 2012; Machado et al. 2015; Simeonidis and Price 2015; Bergdahl et al. 2015). They are based on different approaches and rationales, thereby leading to distinct solutions. One key issue is the multitude of results, known to be dependent on the specific algorithms and solvers, used. In order to address this problem, a consensus-based approach was developed, following our work on metaanalysis of transcriptomic studies (Caldas et al. 2014). This method is based on running several optimization procedures and analyzing a posteriori the solutions, looking for a consensus. The rationale is to have rankings of hypotheses that may provide confidence in particular sets of proposed genetic alterations from various aspects.

In the case of a single population of microbes (i.e. a monoculture), the genome-scale models of L. lactis and C. glutamicum were extended with the biosynthetic pathways of the four above-mentioned target compounds (Fig. 9). Subsequently, five different optimization methods were applied to the genomescale models (extended with the heterologous pathways): OptKnock (Burgard et al. 2003), MOMA (Segrè et al. 2002), OptGene (Patil et al. 2005), RoboKod (Stanford et al. 2015), and RobustKnock (Tepper and Shlomi 2010), and the consensus ranking was obtained for each strain and compound. The hypotheses were generated with the rank product test and the outputs were lists of gene deletions that would achieve the best ranks using defined criteria, namely maximum predicted target compound production, maximizing the minimum predicted target compound production and distance from the wild-type flux distribution. These results were then further filtered and experimentally validated, resulting in several promising mutant strains where production was increased by up to three-fold (unpublished results). Implementation of this pipeline is possible via a software package called OptPipe, available at https:// github.com/AndrasHartmann/OptPipe.

We then explored the concept of multi-objective optimization in the field of metabolic engineering when both continuous and integer decision variables were involved in the model. In particular, we proposed multi-objective models to suggest reaction deletion strategies and to deal with problems where several functions must be optimized simultaneously, such as maximization of bioproducts while minimizing 


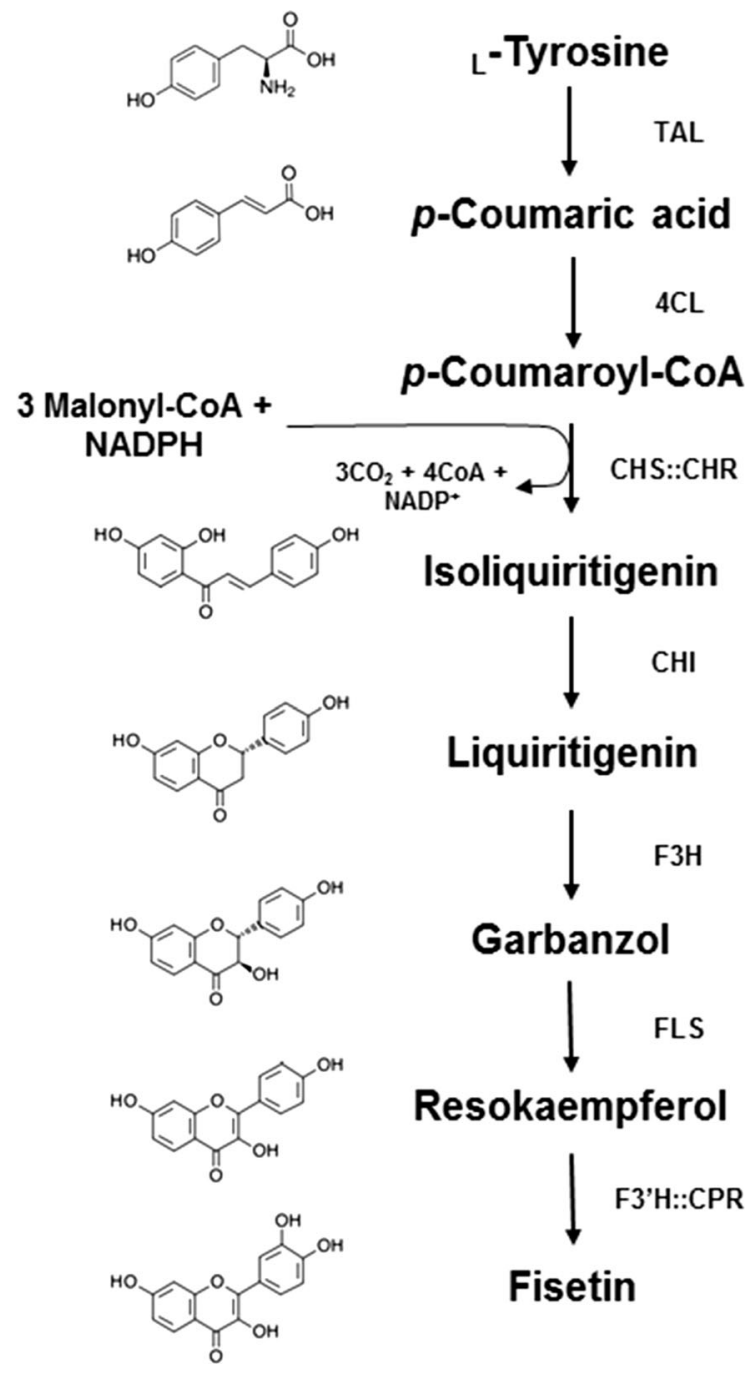

Fig. 8 Heterologous biosynthetic pathway for fisetin assembled in E. coli. TAL tyrosine ammonia-lyase, 4CL 4-coumaroylCoA ligase, $C H S:: C H R$ chalcone synthase::chalcone reductase fusion, $\mathrm{CHI}$ chalcone isomerase, $\mathrm{F} 3 \mathrm{H}$ flavanone 3-hydroxylase, $F L S$ flavonol synthase, $F 3^{\prime} H:: C P R$ flavonoid $3^{\prime}$-hydroxylase::cytochrome $\mathrm{P} 450$ reductase fusion

toxicity. We thus introduced Multi-Objective Mixed integer Optimization for metabolic engineering, a computational framework that aims to model and solve optimization problems, generated for predicting reaction knockout strategies by means of multiobjective programming. We compared the results from our method with those obtained by using the well-known bi-level optimization model (OptKnock). Furthermore, we studied two multi-objective optimization problems arising from the metabolic engineering of microorganisms, showing that indeed a multi-objective setting is promising, as it expands the set of generated hypotheses and allows inclusion of multiple goals, simultaneously.

\section{For a community of microbes}

Pure microbial cultures have long been used for production of high-value compounds, as illustrated by the example of $S$. cerevisiae producing artemisinic acid, an important precursor for the anti-malaria drug artemisinin, on a large scale (Ro et al. 2006; Lenihan et al. 2008). However, there is increasing evidence that such processes could run more efficiently if a community of microbial species is used (Logan and Rabaey 2012; Jones et al. 2016; Wen et al. 2016). There are multiple reasons including an increased production efficiency of the system due to lessening of metabolic burden for individual cells. Another important consideration is that such community systems might have higher chances of avoiding toxic effects specific for individual microorganisms. Microbial consortia are believed to be able to sustain more complex production pathways, as well as being more robust as a group. The challenge, however, remains in establishing precisely which set of strains or species is best for the production of any given compound.

As part of BacHBerry, we introduced an initial topological model and a combinatorial algorithm that enabled us to propose optimal consortia to produce compounds that were either exogenous to the consortium, or were endogenous but where interaction between the species in the consortium could improve the production line (Julien-Laferrière et al. 2016). For initial validations of the model and the method, we applied it to two case-studies taken from the literature. The first case involved production of two bioactive compounds, penicillin and cephalosporin $\mathrm{C}$, both of which are antibiotics used in the pharma industry. A synthetic consortium composed of four different species, three Actinobacteria (Streptomyces cattleya, Rhodococcus jostii RAH_1 and Rc. erythropolis BG43) and one methanogenic Archaea (Methanosarcina barkeri), was then run through the algorithm, allowing introduction of genetic manipulations and regulatory processes. We showed that the best solution involved only two of the four species, namely St. cattleya and M. barkeri (Julien-Laferrière et al. 2016). 
Fig. 9 Heterologous biosynthetic pathways introduced into the genomescale models of $C$. glutamicum and L. lactis leading to the four target compounds: (i) transresveratrol (ii) pelargonidin (iii) quercetin and (iv) fisetin. Two heterologous enzymes (TAL and 4CL) are common for the biosynthesis of the phenolic molecules. Enzyme abbreviations: TAL tyrosine ammonia-lyase, $4 C L$ 4-coumaroyl-CoA ligase, STS stilbene synthase, $C H S$ chalcone synthase, $\mathrm{CHR}$ chalcone reductase, $\mathrm{CHI}$ chalcone isomerase, $\mathrm{F} 3 \mathrm{H}$ flavanone 3-hydroxylase, $F L S$ flavonol synthase, $F 3^{\prime} H$ flavonoid $3^{\prime}$ hydroxylase, CPR cytochrome $\mathrm{P} 450$ reductase

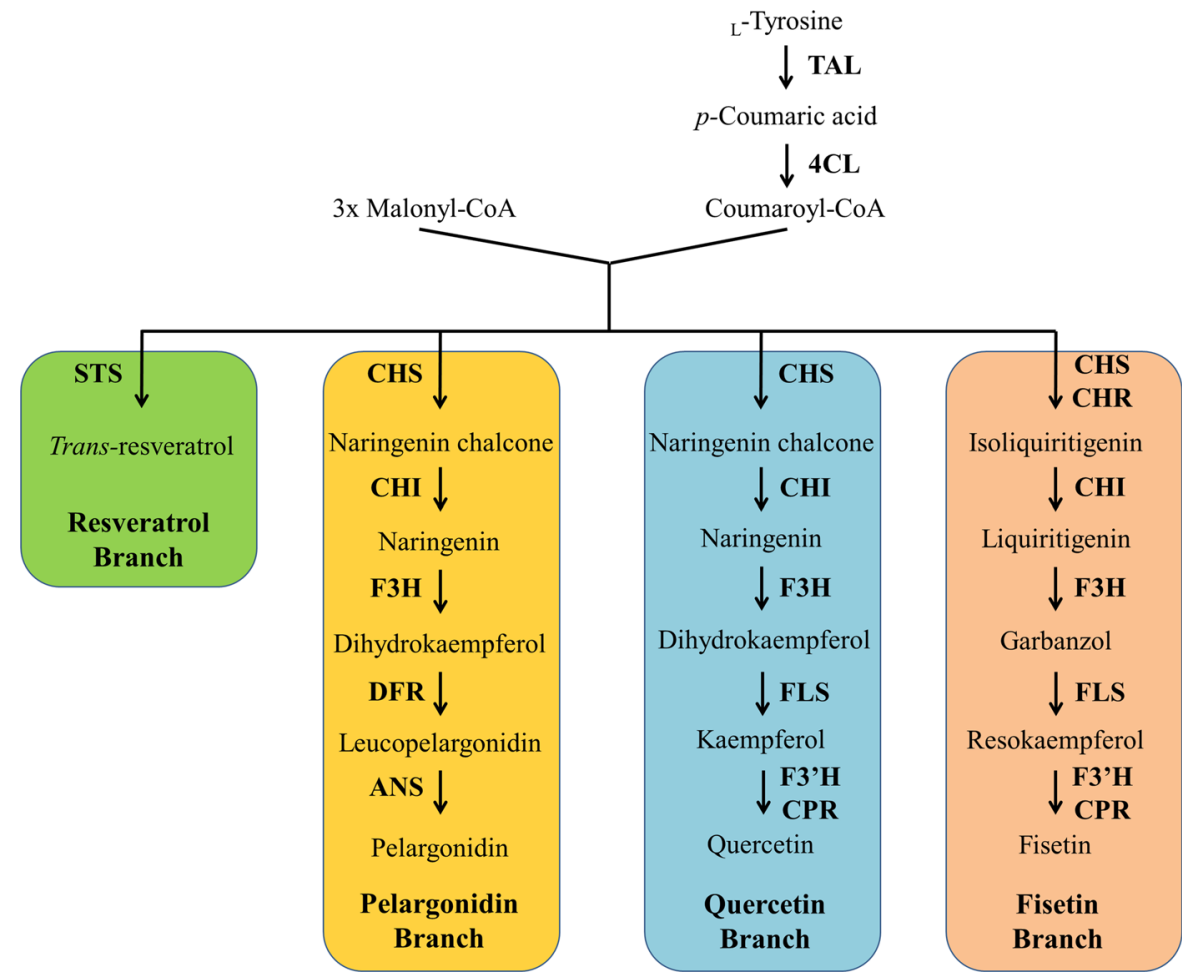

Another considered case was a consortium that may be seen as artificial in the sense that it associates two wild-type populations of microbes, pathogenic $\gamma$ proteobacteria Klebsiella pneumoniae and methanogenic Archaea M. mazei, which normally do not interact in nature. K. pneumoniae is a natural producer of 1,3-propanediol, which is an important building block for polymer production. Our results demonstrated that co-cultivation of $K$. pneumoniae with $M$. mazei may enable obtaining higher yields of this compound in Klebsiella (Julien-Laferrière et al. 2016). This supported a previous study involving co-cultures of Clostridium sp. with methanogenes such as Methanosarcina sp. CHTI55 (Koesnandar et al. 1990). The developed algorithms are currently being used for developing consortia of polyphenol-producing Gram-positive bacteria. This task was inspired by the work of Jones and colleagues, who were able to achieve an overall 970-fold improvement in titers for flavan-3-ols production from phenylpropanoic acids by using a co-culture of two $E$. coli strains with further optimization of cultivation conditions, as compared to previous studies that used monocoltures for this purpose (Jones et al. 2016).

\section{Recovery and purification of polyphenols from fermentation broth}

Downstream processing is an important part of any production process and consists of steps required for recovery and purification of biosynthetic products from fermentation broth, which may have a significant impact on the final costs of a product (Goldberg 1997). In the design of downstream processing within BacHBerry, trans-resveratrol (Fig. 7) was used as the test product, being one of the model phenolic compounds that was successfully produced in both $C$. glutamicum and L. lactis.

In a typical fermentation broth, most of the components present are either hydrophilic (e.g., salts, organic acids, non-consumed carbon sources (usually glucose), nitrogen source (ammonium sulfate, for instance)) or are much larger molecules than transresveratrol (e.g., proteins, nucleic acids, cells in suspension). This suggested a downstream processing design strategy that used either solubility and hydrophobicity as the driving forces, or alternatively-size exclusion. Since, at low concentrations (around $100 \mathrm{mg} \mathrm{l}^{-1}$ ), crystallization would involve high energy costs per $\mathrm{kg}$ of product recovered, this 
option was discarded. Two possible extraction techniques, namely liquid-liquid extraction and solidliquid adsorption, were investigated as means of recovering and purifying extracellularly produced trans-resveratrol from a C. glutamicum fermentation broth containing spent CGXII medium and glucose, due to the higher production efficiency found in this bacterium. The downstream process design aimed at a recovery of at least $80 \%$ and a purity of $95 \%$ transresveratrol.

Liquid-liquid extraction is a technique to separate a given compound from a feed solution using an immiscible auxiliary phase that should be selective for that compound (Cox and Rydberg 2004). The logarithm (base 10) of the partition coefficient of trans-resveratrol between the aqueous and different potential organic phases was determined experimentally for several different solvents (Fig. 10). The spent fermentation broth from C. glutamicum was used as the aqueous phase in these experiments.

All tested organic solvents performed very well in extracting trans-resveratrol (minimum $\log P$ value is $\sim 0.5$, corresponding to a partition coefficient of 3.2), identifying them as suitable candidates for the recovery of this compound (when having different impurities present; Fig. 10).

One of the drawbacks of using liquid-liquid extraction, however, is that it may interfere with protein folding and stability (Prince and Smith 2004). Besides measuring the partition of the polyphenols of interest in the aqueous and organic phases, the same analysis was performed for the total protein content. Although a considerable part of the total protein remained in the aqueous phase, some of the protein seemed to be present in between the two liquid phases (probably as a result of denaturation and foaming; Fig. 11). This phenomenon could potentially reduce the recovery of the desired phenolics, which was also observed experimentally (data not shown).

Solid-liquid adsorption using hydrophobic resins is another way of purifying trans-resveratrol. As in liquid-liquid extraction, the high hydrophobicity of trans-resveratrol can be used to recover it selectively from the fermentation broth, leaving the more hydrophilic impurities behind. After an initial resin screen, one of the resins selected was the polystyrenedivinylbenzene based Amberlite XAD-16 from Sigma-Aldrich (particle diameter: 250-841 $\mu \mathrm{m}$; dipole moment: 0.3; surface area: $900 \mathrm{~m}^{2} \mathrm{~g}^{-1}$; pore diameter: $100 \AA$ ).

To test the ability of this resin to recover transresveratrol from C. glutamicum fermentation broth (containing CGXII spent medium and glucose), a labscale experiment was performed with an AKTA Explorer chromatographic workstation (GE Healthcare, Sweden; see Fig. 12). A $30 \mathrm{ml}$ column was packed with resin and pre-equilibrated with MilliQ water. Afterwards, $10 \mathrm{ml}$ of sample was injected and a washing step with pure water was performed for 10 column volumes $(\mathrm{CV})$. During elution, a gradient of water/ethanol was run for about $12 \mathrm{CV}$ and the peak of trans-resveratrol showed up at around $18 \mathrm{CV}$, at an ethanol percentage of $50 \%(\mathrm{v} / \mathrm{v})$. Regeneration was performed with $100 \%$ ethanol.

More hydrophilic molecules such as organic acids, sugars and salts appeared in the flow-through (the first $10 \mathrm{CV}$ ). During the water/ethanol gradient, molecules desorbed as a function of their hydrophobicity. More hydrophobic molecules, such as trans-resveratrol, are supposed to elute at higher ethanol content in the mobile phase. In this case, trans-resveratrol appeared to be the compound eluting between 16 and $20 \mathrm{CV}$, since it also showed absorption at $304 \mathrm{~nm}$ (Camont et al. 2009).

Although both liquid-liquid extraction and solidliquid adsorption present their own advantages and disadvantages, a strong point for using adsorption is the use of ethanol instead of any other organic solvent that might not be regarded as GRAS. Moreover, not using liquid-liquid extraction avoids any possible problems related with protein precipitation and emulsification. The ethanol used during elution in adsorption needs to be recovered from water by distillation (the azeotrope poses no problem as $80 \%(\mathrm{v} / \mathrm{v})$ ethanol can be used).

The proposed downstream process started with a solid-liquid separation step by centrifugation to separate biomass from the liquid broth. The clarified liquid phase was then sent continuously to one of two adsorption columns, operating staggered in time. The fraction rich in trans-resveratrol was subsequently evaporated, resulting in a wet slurry containing the polyphenol in the solid phase. This slurry was filtered in a rotary vacuum filter and washed with water to remove remaining impurities adhered to the surface of the crystals. The final step was a drying step to obtain the product in its final solid form. All the outlet 


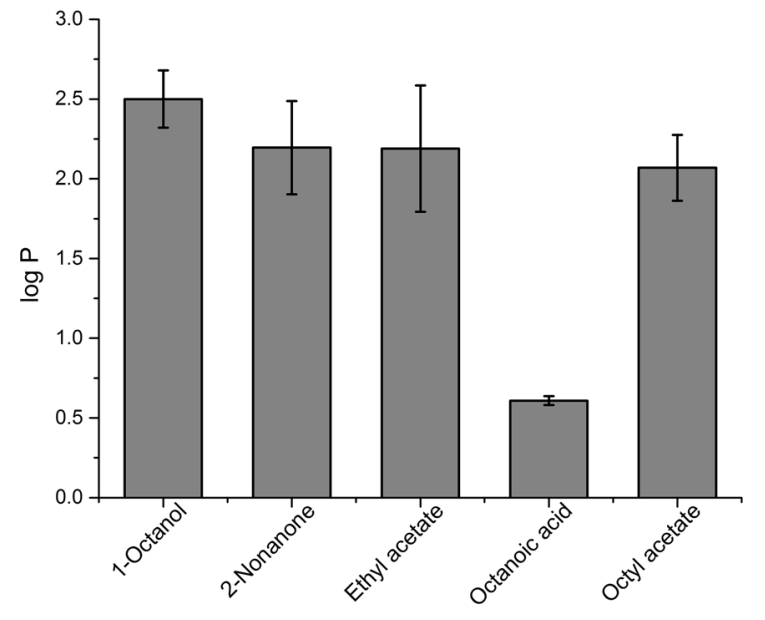

Fig. 10 Partition coefficients in the form of $\log P$ values of trans-resveratrol using different organic solvents using $C$. glutamicum fermentation broth as the aqueous phase

streams containing ethanol were directed to a distillation column in order to recover the solvent. A makeup stream ethanol was added to compensate for losses.

\section{Optimization of phenolic production by fermentation and scale-up of bioprocesses}

There are multiple differences between microbial cultivation in shake flasks versus in bioreactors. The former lacks monitoring and control of various parameters, such as dissolved $\mathrm{O}_{2}$ concentration and pH (Zelle et al. 2010). Furthermore, the fed-batch fermentation mode, where one or more nutrient is being supplied throughout cultivation (Moulton 2014), can be done in a controllable and reproducible fashion only in bioreactors. These properties make bioreactors a suitable stage for optimization of cultivation conditions, often leading to significant improvements in production yields (Hujanen et al. 2001; Ratnam et al. 2003; Li et al. 2010; Rani and Appaiah 2011).

From the beginning of the project it was clear that optimization of fermenter operation would need to be carried out using a model compound, because any newly-identified compounds would only be available at the end of the project. Hence this section of the project was focused on optimization of production of the model compound trans-resveratrol. However, it was expected that the strategies defined and the conclusions extracted from these results on transresveratrol will be valid for the optimization of the fermentation processes for the production of other target compounds. Several L. lactis and C. glutamicum strains designed within the project were evaluated and used for fermenter process optimization. The experiments were carried out in agitated 2-5 1 fermenters with automatically-controlled $\mathrm{pH}$, dissolved oxygen

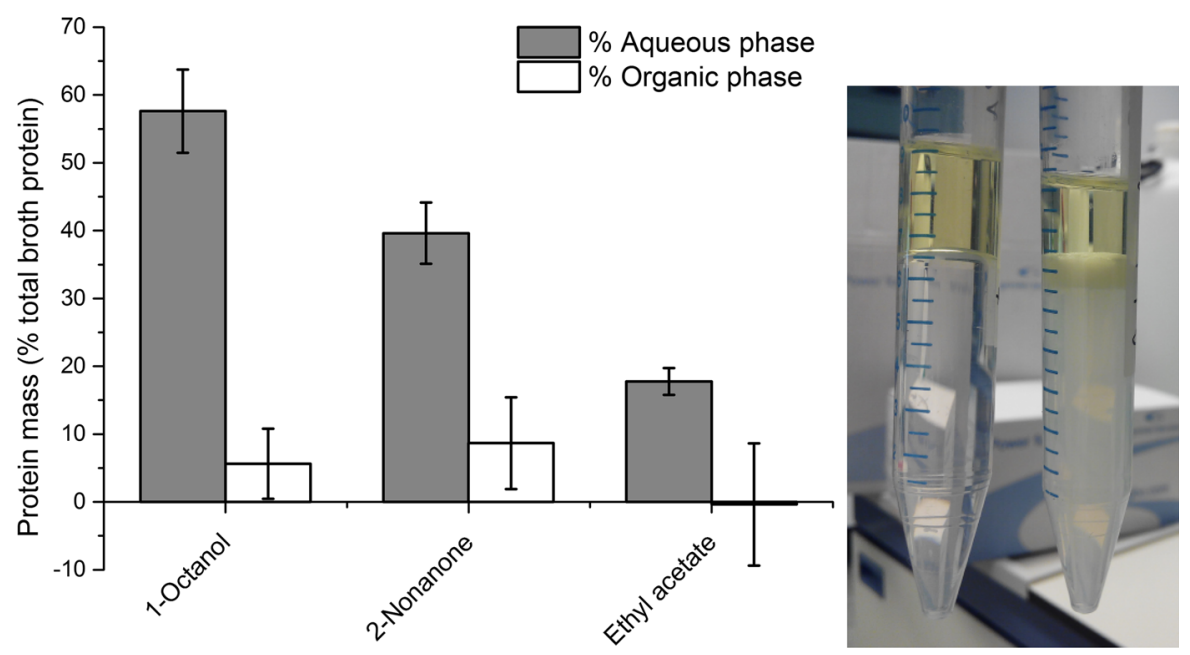

Fig. 11 On the left, protein concentration (expressed as percentage of the initial broth protein concentration) in the organic and aqueous phases, after performing liquid extraction. The concentration in the organic phase is almost zero (considering the error bars), indicating that some protein stayed in the interphase. On the right, the formation of an interphase after the broth had been extracted with organic solvent (a mixture of heptanoic acid and hexyl acetate in this case) was clear 


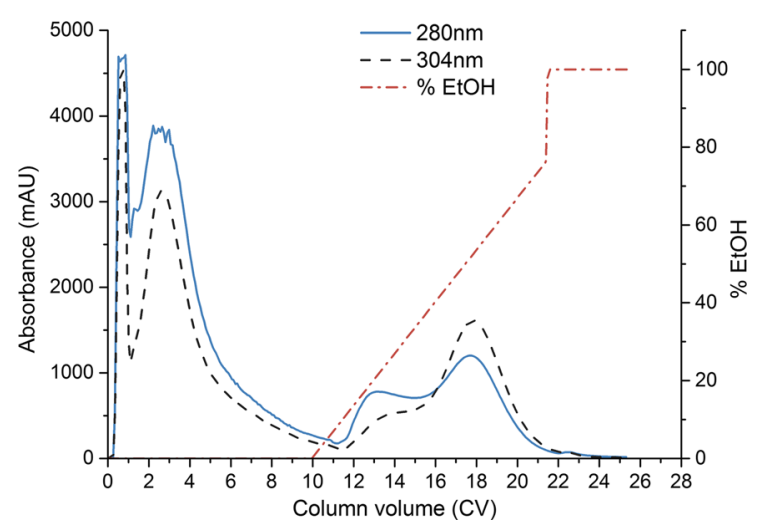

Fig. 12 Chromatogram following a pulse injection of $10 \mathrm{ml}$ of C. glutamicum spent fermentation broth in a $30 \mathrm{ml}$ column packed with Amberlite XAD-16 resin. A washing step was performed with 10 column volumes (CVs) of MilliQ water. Afterwards, elution was performed with a water/ethanol gradient for $12 \mathrm{CV}$, followed by a final regeneration step with $100 \%$ ethanol

and temperature. Whenever required, oxygen was transferred to the medium by air injection at the bottom of the vessel.

A major goal of the project was the development of an economically feasible and sustainable industrial process, therefore the following key optimization criteria were defined: (a) minimization of the operation and investment cost and (b) maximization of the production rate and operation yield. To achieve these optimization goals, the following variables have been investigated: (1) fermentation medium composition, (2) dissolved oxygen concentration, (3) operation strategy (batch vs. fed-batch and integrated vs. separate downstream operation). Our experiments suggested that there were several constraints limiting the operation strategy to be implemented, namely: cell metabolism, the stability of trans-resveratrol inside the fermenter, and the affinity of trans-resveratrol for the adsorption medium and solvents compared to other compounds present in the fermentation broth.

Medium composition is an important parameter that often has a significant contribution towards the final production costs. According to our initial experiments, supplementation of $C$. glutamicum minimal medium with Brain-Heart Infusion (BHI) and a fattyacid biosynthesis inhibitor, cerulenin, had a strong positive effect on the final titers of trans-resveratrol. However, removing these two components from the medium reduced medium costs by approximately 300 -fold, resulting in a more than 50-fold drop in production costs $(267 € / \mathrm{g}$ compared to $5 € / \mathrm{g}$ of transresveratrol without supplementation of $\mathrm{BHI}$ and cerulenin).

We also evaluated the feasibility of producing phenolic compounds from waste materials, namely cheese whey using L. lactis as a host. Whey is a lactose-rich dairy industry by-product with a high environmental impact, if dumped untreated (Palmieri et al. 2017). To achieve this task, our production strain was modified to allow it to grow and produce transresveratrol from lactose by introducing a plasmid carrying the lac operon (Maeda and Gasson 1986). Production at 2 litre-scale was tested using lactose as carbon source and comparing the productivity between the cultures with glucose or lactose. These experiments demonstrated that the production of trans-resveratrol from lactose, and therefore from whey, is possible with slightly inferior productivity compared to glucose. However, if whey is chosen as the fermentation medium, other, more important, issues arise. The cost of whey is comparable to the cost of glucose but the cost of the fermentation medium is only a small part of the total production costs, so variables such as whey availability and quality, as well as the stability of supply and collecting logistics will determine the feasibility of the operation more than the small losses in productivity. Clearly, if a phenolic production industrial unit were to be implemented inside a dairy company with excess whey, the whey might then be the best raw material.

Another parameter investigated was the influence of dissolved oxygen concentration on the production efficiency of L. lactis. This bacterium can activate its respiratory chain in the presence of hemin in the culture medium, and this has been demonstrated to enhance its survival allowing it to grow to higher cell densities (Duwat et al. 2001). Therefore the production strain efficiency was tested under the following conditions: aerobic with hemin added to the fermentation broth, aerobic without hemin, semi-aerobic, and fully anaerobic. In line with the available data, there was a significant increase in the final biomass when the strain was grown under aerobic conditions with hemin. However, the opposite was observed for the production of trans-resveratrol where the highest amounts of the compound were detected under anaerobic conditions. 


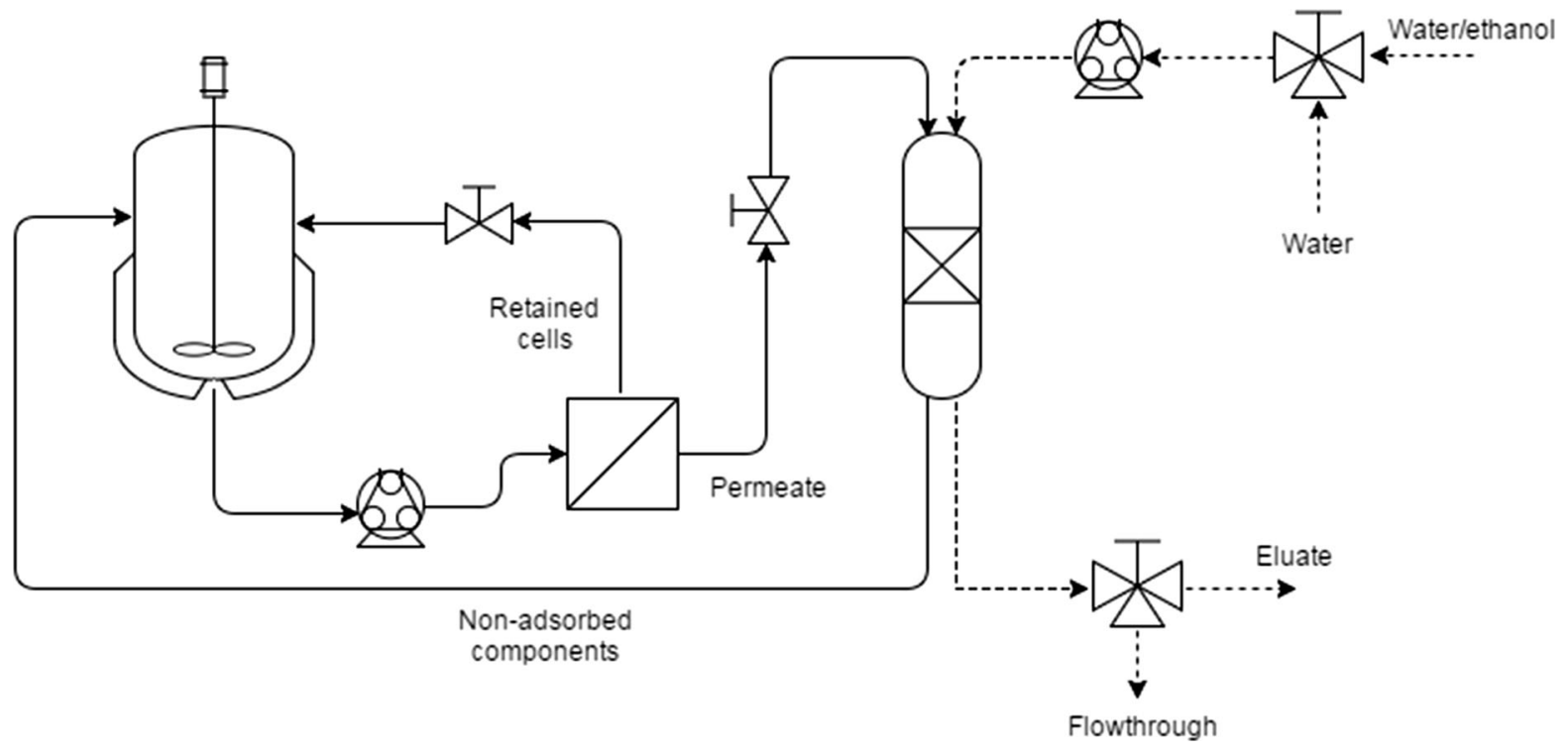

Fig. 13 Experimental set-up for the "continuous product removal" mode

Lastly, we have also worked on establishing a system for continuous product removal from the fermentation broth in order to simplify downstream processing and reduce trans-resveratrol oxidation due to prolonged incubation. Two strategies for continuous adsorption and storage of secreted trans-resveratrol in a resin have been devised and tested: (1) "in situ" product removal that consisted of adding a resin directly to the fermentation broth and (2) "continuous product removal" (ConPR) that involved constant pumping of the fermentation broth through a column filled with the adsorption resin. The supernatant was separated from cells via a tangential flow micro-filtration device, sent though the column, and then returned back into the fermenter (Fig. 13).

The obtained results demonstrated that both the "in situ" and ConPR strategies lowered the transresveratrol productivity, probably due to co-adsorption of $p$-coumaric acid, the precursor for transresveratrol. This effect was most pronounced in the "in situ" operation mode due to continuous contact of the resin with the supernatant and cells. In the ConPR operation mode the effect was reduced due to low supernatant flow rate through the resin, however adsorption of trans-resveratrol was also reduced. In contrast, in the "in situ" mode the amount of adsorbed end-product was nearly identical to the amount of extracellular and intracellular trans-resveratrol produced in control experiments without addition of resin. Although the two techniques did not increase the overall productivity, they still simplified the downstream processing and may lead to a production cost reduction by eliminating the need to break down the cells. However, future research may provide a solution to avoid precursor co-adsorption resulting in increased polyphenol productivity.

\section{Dissemination, training, societal and ethical issues}

In addition to applying the latest technological advances from life sciences for establishing a pipeline for microbial-based polyphenol production, BacHBerry analyzed the economic potential of the possible products developed from this research. The assessment of economic issues of the project has been focused on the market potential of the natural colorants identified during the screening of the germplasm collection. This was done along with obtaining an overview of the relevant regulations and procedures for approval of novel food additives, including colorants, in the EU, the United States (US), and China. Regulations within both the EU and China confer a strict set of rules for bringing a novel 
Table 5 Summary of the outcomes of the project

\begin{tabular}{|c|c|c|c|c|}
\hline $\begin{array}{l}\text { Specific } \\
\text { application } \\
\text { area in } \\
\text { project }\end{array}$ & $\begin{array}{l}\text { Bioprospecting for } \\
\text { discovery of novel berry } \\
\text { phenolics }\end{array}$ & $\begin{array}{l}\text { Characterization of } \\
\text { phenolics biosynthetic } \\
\text { pathways }\end{array}$ & $\begin{array}{l}\text { Bacterial cell factories for } \\
\text { production of berry } \\
\text { phenolics }\end{array}$ & $\begin{array}{l}\text { Fermentation and } \\
\text { bioprocess engineering }\end{array}$ \\
\hline $\begin{array}{l}\text { General aims } \\
\text { of } \\
\text { BacHBerry }\end{array}$ & $\begin{array}{l}\text { To tap into the biodiversity of } \\
\text { berries from around the } \\
\text { globe and of their phenolics } \\
\text { content }\end{array}$ & $\begin{array}{l}\text { Identification and } \\
\text { characterization of berry } \\
\text { phenolics biosynthetic } \\
\text { pathways }\end{array}$ & $\begin{array}{l}\text { Design and generation of } \\
\text { bacterial cell factories for } \\
\text { production of high-value } \\
\text { berry phenolics }\end{array}$ & $\begin{array}{l}\text { Implementation of } \\
\text { production of value-added } \\
\text { phenolics in fermenters up } \\
\text { to demonstration scale }\end{array}$ \\
\hline $\begin{array}{l}\text { Achievements } \\
\text { of the } \\
\text { project }\end{array}$ & $\begin{array}{l}\text { Standardization of } \\
\text { methodologies for } \\
\text { harvesting, extract } \\
\text { preparation, phenolics } \\
\text { content assessment and } \\
\text { fractionation } \\
\text { Metabolomics data from } \\
\text { berry species originating } \\
\text { from UK, mainland Europe, } \\
\text { Russia, Chile, and China } \\
\text { was obtained and made } \\
\text { available in form of an } \\
\text { online database } \\
\text { Implementation of robust } \\
\text { assays based on the } \\
\text { SMART platform for } \\
\text { discovery of bioactivity and } \\
\text { functionality } \\
\text { Obtained berry extracts were } \\
\text { assayed for bioactivity } \\
\text { against several human } \\
\text { diseases, and additionally } \\
\text { evaluated for other } \\
\text { functional uses (eq. food } \\
\text { additives, antimicrobials). } \\
\text { Bioactivities against } \\
\text { Alzheimer's, Parkinson's } \\
\text { and Huntington's diseases, } \\
\text { Amyotrophic Lateral } \\
\text { Sclerosis and inflammation } \\
\text { were detected } \\
\text { Several pure bioactive } \\
\text { effector compounds were } \\
\text { identified and validated }\end{array}$ & $\begin{array}{l}\text { Transcriptomes of } 13 \\
\text { germplasms spanning } \\
\text { eight genera, seven } \\
\text { families and seven } \\
\text { orders were generated } \\
\text { and analyzed } \\
\text { New algorithms for } \\
\text { functional genomics and } \\
\text { improved computational } \\
\text { methods for pathway } \\
\text { identification were } \\
\text { established } \\
\text { Over } 4000 \text { candidate } \\
\text { genes for various } \\
\text { biosynthetic steps, } \\
\text { transport and regulation } \\
\text { of polyphenol } \\
\text { production were } \\
\text { identified } \\
\text { Biochemical activities of } \\
\text { some of the gene } \\
\text { candidates were tested, } \\
\text { thus validating the } \\
\text { predictions }\end{array}$ & $\begin{array}{l}\text { Lactococcus lactis strains } \\
\text { were engineered for } \\
\text { stilbene (trans-resveratrol } \\
\text { and pterostilbene) and } \\
\text { anthocyanin production } \\
\text { Production of various } \\
\text { stilbenes (trans-resveratrol, } \\
\text { pinosylvin, and } \\
\text { piceatannol), methylated } \\
\text { stilbenes (pinostilbene and } \\
\text { pterostilbene), flavanones } \\
\text { (naringenin, pinocembrin, } \\
\text { and eryodictiol), and } \\
\text { flavonols (kaempferol and } \\
\text { quercetin) was achieved in } \\
\text { C. glutamicum with yields } \\
\text { comparable to those } \\
\text { obtained in the model } \\
\text { organism E. coli. } \\
\text { Production titers for the } \\
\text { produced flavonols are the } \\
\text { highest titers obtained in } \\
\text { engineered microorganism } \\
\text { until today } \\
\text { Polyphenol production was } \\
\text { further improved by } \\
\text { engineering enhanced } \\
\text { precursor supply (eq. } \mathrm{L}^{-} \\
\text {tyrosine, malonyl-CoA) via } \\
\text { rational design, modeling- } \\
\text { based prediction, and use of } \\
\text { biosensors } \\
\text { Alternative biosynthetic } \\
\text { routes were explored } \\
\text { though engineering of } \\
\text { trans-resveratrol } \\
\text { production from } \\
\text { 4-hydroxybenzoic acid } \\
\text { (HBA) in C. glutamicum } \\
\text { predicting a minimal set of } \\
\text { modifications needed for } \\
\text { improved polyphenol } \\
\text { production in both a single- } \\
\text { strain population and a } \\
\text { consortium of two or more } \\
\text { species }\end{array}$ & $\begin{array}{l}\text { Cultivation conditions for } \\
\text { improved productivity were } \\
\text { developed for both } L \text {. lactis } \\
\text { and C. glutamicum through } \\
\text { optimization of } \\
\text { fermentation parameters } \\
\text { (batch vs. fed-batch } \\
\text { operation, substrate and } \\
\text { dissolved oxygen } \\
\text { concentration, etc.) } \\
\text { Whey was evaluated as an } \\
\text { alternative carbon source } \\
\text { for polyphenol production } \\
\text { in } L \text { lactis } \\
\text { Multiple bioseparation } \\
\text { methods for extraction of } \\
\text { produced polyphenols from } \\
\text { fermentation broth were } \\
\text { evaluated } \\
\text { Two possible designs for a } \\
\text { system for continuous } \\
\text { product removal from the } \\
\text { fermentation broth were } \\
\text { implemented } \\
\text { Resveratrol production in } C \text {. } \\
\text { glutamicum was scaled up } \\
\text { from shake flask to } 51 \\
\text { fermenter to demonstration } \\
\text { scale ( } 250 \text { 1) }\end{array}$ \\
\hline
\end{tabular}


food colorant, even a natural one, to the market. These rules consider not only the composition and purity of the substance, but also the process through which the compounds have been produced. In contrast, in the US, a new food additive can gain access to the market by simply passing the GRAS assessment. Hence, if a novel polyphenolic colorant could be produced using engineered microbial cell factories, the US market should probably be the first market to be targeted, owing to less strict regulatory requirements for the approval.

Another topic addressed in BacHBerry was access and benefit sharing of genetic resources. As the project was built upon screening of berry species from multiple countries, including China, Russia, Chile, Portugal, and the UK, it is important to harness these resources in a responsible manner. The Nagoya Protocol on Access to Genetic Resources and the Fair and Equitable Sharing of Benefits Arising from their Utilization (NP-ABS) is an agreement consisting of a set of rules for fair sharing of monetary and nonmonetary benefits arising from the usage and commercialization of genetic resources and traditional knowledge associated with those. It aims at achieving fairness and equity of gain distribution, especially towards indigenous and local communities (SCBD 2011; Morgera et al. 2014). The Protocol came into force in the EU on June 9th 2014. As most of the BacHBerry project partners were from EU Member States, we began collecting information about the best practices suggested from both the Convention on Biological Diversity (CBD) and the EU, and kept monitoring the relevant guidelines and updates from the EU on implementing ABS. Among the home countries of the project partners China, Denmark, France, Germany, Switzerland, and UK ratified or accepted the NP-ABS (as of November 2016) and began implementing the aforementioned regulations (European Parliament 2014). The UK also made its own regulation on implementing the Protocol (NMRO (UK) 2015; Secretary of State (UK) 2015).

Funded under the theme of "Knowledge-based BioEconomy", a final aim of the project was to provide scientific and technical solutions to harness berry resources, as well as to contribute to sustainable development of the economy. We consequently investigated the possible contributions of our research to build a more sustainable society based on environmental, economical, and social impacts (Pei and
Schmidt 2016). Furthermore, we conducted several open dialog events, namely workshops and interviews with potential stakeholders from Austria, Denmark, and China. One of the surprising outcomes was that the general public raised concerns over the use of genetically-modified organisms (GMOs) in the pipeline a lot less frequently than originally expected. A free, science-based game for mobile devices called "Berry Maker" was produced (http://www. berrymaker.com). The game allows the user to follow the pipeline designed within our project, from acquisition of diverse berries, to extraction and analysis of biosynthetic genes, to microbial fermentationbased production of specific polyphenols and running a biotech factory that is exposed to real world situations, such as price fluctuations. The game is also available on iTunes ${ }^{1}$ and Google Play ${ }^{2}$ store. Lastly, a 22 min documentary film was produced, showing sampling of berries, analysis of interesting compounds, and experiments with natural color pigments for food products from across Europe (see: https:// vimeo.com/193467652).

\section{Conclusions}

During 3 years of the project, the BacHBerry partners successfully established a complete pipeline for screening, bioprospecting, identification, and microbial production of plant phenolic compounds in Grampositive bacteria. A summary of the most important results is presented in Table 5. The major highlights are construction of the extensive germplasm collection database, establishment of a method for high-throughput screening of bioactive extracts using the yeastbased human disease models, generation of a vast berry transcriptome database, construction of microbial cell factories in L. lactis and C. glutamicum for the production of polyphenols with yields comparable to those currently available from the literature, release of several software packages for transcriptome analysis and in silico-based metabolic engineering, as well as optimization of fermentation conditions and downstream processing for the selected organisms aimed at

\footnotetext{
${ }^{1}$ See: https://itunes.apple.com/us/app/berrymaker-dna-sodafactory/id $1163115116 ? \mathrm{~s}=1 \& \mathrm{mt}=8$.

${ }^{2}$ See: https://play.google.com/store/apps/details?id=com. biofaction.berrymaker.
} 
increasing the yield and the purify of the final products. The pipeline has been completely validated and is available to be picked up by the biotech industry for further improvement and commercialization. The developed methods and protocols should be suitable for discovery, as well as production of most groups of polyphenols in the above-mentioned organisms, without a need for significant alterations and adaptations. Overall, this project provided the scientific community with enabling tools and resources to further advancing research on berries, phenolic compounds, and metabolic engineering of microorganisms. Additional efforts were also focused on communication with stakeholders, public outreach, and popularization of science among the younger generation. The majority of the project outcomes is already available to the scientific community and the industry in form of peer-reviewed articles, or will be published within the coming year.

Acknowledgements The authors would like to thank the European Union's Seventh Framework Programme (BacHBerry, Project No. FP7-613793, and FP7-PEOPLE2013-COFUND, Project No. FP7-609405) for the financial support. AD, RF, MHHN, PG, SGS, and JF would also like to acknowledge the Novo Nordisk Foundation. We express our gratitude to Dr. Martha Cyert (Stanford School of Medicine, EUA), Dr. Hitoshi Shimoi (National Research Institute of Brewing, Japan) and Dr. Yoshio Araki (Graduate School of Biosphere Science, Hiroshima University, Japan) for providing the yeast strain YAA5. We also thank Dr. Ian Macraedie, RMIT University, Australia) for providing the plasmid p416_GPDprGFP-A42.

\section{References}

Andrade R, Wannagat M, Klein CC et al (2016) Enumeration of minimal stoichiometric precursor sets in metabolic networks. Algorithms Mol Biol 11:25. doi:10.1186/s13015016-0087-3

Aranaz I, Acosta N, Mengíbar MN et al (2016) InFiQuS: making the best of leftovers. In: de María PD (ed) Industrial biorenewables: a practical viewpoint. Wiley, Hoboken, pp 363-364

Bak S, Olsen CE, Halkier BA, Møller BL (2000) Transgenic tobacco and Arabidopsis plants expressing the two multifunctional sorghum cytochrome P450 enzymes, CYP79A1 and CYP71E1, are cyanogenic and accumulate metabolites derived from intermediates in Dhurrin biosynthesis. Plant Physiol 123:1437-1448. doi:10.1104/pp.123.4.1437

Becker J, Wittmann C (2012) Bio-based production of chemicals, materials and fuels-Corynebacterium glutamicum as versatile cell factory. Curr Opin Biotechnol 23:631-640. doi:10.1016/j.copbio.2011.11.012

Becker J, Armstrong G, Vandermerwe M et al (2003) Metabolic engineering of for the synthesis of the wine-related antioxidant resveratrol. FEMS Yeast Res 4:79-85. doi:10. 1016/S1567-1356(03)00157-0

Beekwilder J, Wolswinkel R, Jonker H et al (2006) Production of resveratrol in recombinant microorganisms. Appl Environ Microbiol 72:5670-5672. doi:10.1128/AEM. 00609-06

Bergdahl B, Sonnenschein N, Machado D et al (2015) Genomescale models. In: Villadsen J (ed) Fundamental bioengineering. Wiley-VCH Verlag GmbH \& Co. KGaA, Weinheim, pp 143-182

Bhan N, Xu P, Khalidi O, Koffas MAG (2013) Redirecting carbon flux into malonyl-CoA to improve resveratrol titers: proof of concept for genetic interventions predicted by OptForce computational framework. Chem Eng Sci 103:109-114. doi:10.1016/j.ces.2012.10.009

Bharadwaj P, Martins R, Macreadie I (2010) Yeast as a model for studying Alzheimer's disease. FEMS Yeast Res 10:961-969. doi:10.1111/j.1567-1364.2010.00658.x

Blomstedt CK, Gleadow RM, O'Donnell N et al (2012) A combined biochemical screen and TILLING approach identifies mutations in Sorghum bicolor L. Moench resulting in acyanogenic forage production. Plant Biotechnol J 10:54-66. doi:10.1111/j.1467-7652.2011. 00646.x

Bodvard K, Jörhov A, Blomberg A et al (2013) The yeast transcription factor Crzl is activated by light in a $\mathrm{Ca} 2+/$ calcineurin-dependent and PKA-independent manner. PLoS ONE 8:e53404. doi:10.1371/journal.pone. 0053404

Borrill P, Ramirez-Gonzalez R, Uauy C (2016) expVIP: a customizable RNA-seq data analysis and visualization platform. Plant Physiol 170:2172-2186. doi:10.1104/pp.15. 01667

Burgard AP, Pharkya P, Maranas CD (2003) Optknock: a bilevel programming framework for identifying gene knockout strategies for microbial strain optimization. Biotechnol Bioeng 84:647-657. doi:10.1002/bit.10803

Burkovski A (ed) (2008) Corynebacteria: genomics and molecular biology. Caister Academic Press, Poole

Caldas J, Vinga S, Nordmann A et al (2014) Global metaanalysis of transcriptomics studies. PLoS ONE 9:e89318. doi:10.1371/journal.pone.0089318

Camont L, Cottart C-H, Rhayem Y et al (2009) Simple spectrophotometric assessment of the trans-/cis-resveratrol ratio in aqueous solutions. Anal Chim Acta 634:121-128. doi:10.1016/j.aca.2008.12.003

Cao W, Ma W, Zhang B et al (2016) Improved pinocembrin production in Escherichia coli by engineering fatty acid synthesis. J Ind Microbiol Biotechnol 43:557-566. doi:10. 1007/s10295-015-1725-3

Carvalho E, Franceschi P, Feller A et al (2013) A targeted metabolomics approach to understand differences in flavonoid biosynthesis in red and yellow raspberries. Plant Physiol Biochem PPB 72:79-86. doi:10.1016/j.plaphy. 2013.04.001 
Catalgol B, Batirel S, Taga Y, Ozer NK (2012) Resveratrol: French paradox revisited. Front Pharmacol 3:141. doi:10. 3389/fphar.2012.00141

Chaovanalikit A, Thompson MM, Wrolstad RE (2004) Characterization and quantification of anthocyanins and polyphenolics in blue honeysuckle (Lonicera caerulea $\mathrm{L}$.). J Agric Food Chem 52:848-852. doi:10.1021/JF030509O

Chen X, Zhou L, Tian K et al (2013) Metabolic engineering of Escherichia coli: a sustainable industrial platform for biobased chemical production. Biotechnol Adv 31:1200-1223. doi:10.1016/j.biotechadv.2013.02.009

Choi O, Wu C-Z, Kang SY et al (2011) Biosynthesis of plantspecific phenylpropanoids by construction of an artificial biosynthetic pathway in Escherichia coli. J Ind Microbiol Biotechnol 38:1657-1665. doi:10.1007/s10295-011-0954-3

Costa RS, Veríssimo A, Vinga S et al (2014) Ki MoSys: a webbased repository of experimental data for KInetic MOdels of biological SYStems. BMC Syst Biol 8:85. doi:10.1186/ s12918-014-0085-3

Costa RS, Hartmann A, Vinga S (2016) Kinetic modeling of cell metabolism for microbial production. J Biotechnol 219:126-141. doi:10.1016/j.jbiotec.2015.12.023

Cox M, Rydberg J (2004) Introduction to solvent extraction. In: Rydberg J, Cox M, Musikas C, Choppin GR (eds) Solvent extraction principles and practice, 2nd edn. Marcel Decker Inc, New York

Cragg GM, Newman DJ (2013) Natural products: a continuing source of novel drug leads. Biochim Biophys Acta Gen Subj 1830:3670-3695. doi:10.1016/j.bbagen.2013.02.008

Dobson R, Gray V, Rumbold K (2012) Microbial utilization of crude glycerol for the production of value-added products. J Ind Microbiol Biotechnol 39:217-226. doi:10.1007/ s10295-011-1038-0

Duarte K, Rocha-Santos TAP, Freitas AC, Duarte AC (2012) Analytical techniques for discovery of bioactive compounds from marine fungi. TrAC Trends Anal Chem 34:97-110. doi:10.1016/j.trac.2011.10.014

Dumont FJ (2000) FK506, an immunosuppressant targeting calcineurin function. Curr Med Chem 7:731-748

Duwat P, Sourice S, Cesselin B et al (2001) Respiration capacity of the fermenting bacterium Lactococcus lactis and its positive effects on growth and survival. J Bacteriol 183:4509-4516. doi:10.1128/JB.183.15.4509-4516.2001

Dvora H, Koffas MAG (2013) Microbial production of flavonoids and terpenoids. In: McNeil B, Archer D, Giavasis I, Harvey L (eds) Microbial production of food ingredients, enzymes and nutraceuticals. Woodhead Publishing, Oxford, pp 234-261

Eggeling L, Bott M (eds) (2005) Handbook of Corynebacterium glutamicum. CRC Press, Boca Raton

European Parlament (2014) Regulation (EU) no 511/2014 of the European parliament and of the council of 16 April 2014 on compliance measures for users from the Nagoya Protocol on access to genetic resources and the fair and equitable sharing of benefits arising from their utilization in the union. http:// eur-lex.europa.eu/legal-content/EN/TXT/PDF/?uri= CELEX:32014R0511\&from=EN. Accessed 07 Sept 2017

Falcone Ferreyra ML, Rius SP, Casati P (2012) Flavonoids: biosynthesis, biological functions, and biotechnological applications. Front Plant Sci 3:222. doi:10.3389/fpls.2012. 00222

Fernandes A, Vinga S, Kern A et al (2016) Improving protein expression prediction using extra features and ensemble averaging. PLoS ONE 11:e0150369. doi:10.1371/journal. pone. 0150369

Figueira I, Menezes R, Macedo D, et al. (2017) Polyphenols beyond barriers: a glimpse into the brain. Curr Neuropharmacol. 15:562-594. doi:10.2174/1570159X14666 161026151545

Fredes C, Yousef GG, Robert P et al (2014) Anthocyanin profiling of wild maqui berries (Aristotelia chilensis [Mol.] Stuntz) from different geographical regions in Chile. J Sci Food Agric 94:2639-2648. doi:10.1002/jsfa.6602

Frelet-Barrand A, Boutigny S, Moyet L et al (2010) Lactococcus lactis, an alternative system for functional expression of peripheral and intrinsic arabidopsis membrane proteins. PLoS ONE 5:e8746. doi:10.1371/journal.pone.0008746

Funakoshi-Tago M, Nakamura K, Tago K et al (2011) Antiinflammatory activity of structurally related flavonoids, Apigenin, Luteolin and Fisetin. Int Immunopharmacol 11:1150-1159. doi:10.1016/j.intimp.2011.03.012

Garcia G, Santos CND, Menezes R (2016) High-throughput yeast-based reporter assay to identify compounds with antiinflammatory potential. Methods Mol Biol 1449:441-452. doi:10.1007/978-1-4939-3756-1_29

Gaspar P, Carvalho AL, Vinga S et al (2013) From physiology to systems metabolic engineering for the production of biochemicals by lactic acid bacteria. Biotechnol Adv 31:764-788. doi:10.1016/j.biotechadv.2013.03.011

Ghosh D, Scheepens A (2009) Vascular action of polyphenols. Mol Nutr Food Res 53:322-331. doi:10.1002/mnfr. 200800182

Giusti MM, Wrolstad RE (2003) Acylated anthocyanins from edible sources and their applications in food systems. Biochem Eng J 14:217-225. doi:10.1016/S1369703X(02)00221-8

Goldberg E (1997) Handbook of downstream processing. Springer, London

Goszcz K, Deakin SJ, Duthie GG et al (2015) Antioxidants in cardiovascular therapy: panacea or false hope? Front Cardiovasc Med 2:29. doi:10.3389/fcvm.2015.00029

Grand View Research Inc. (2016) Polyphenols market analysis by product (grape seed, green tea, apple), by application (functional food, functional beverages, dietary supplements) and segment forecasts to 2024. http://www. grandviewresearch.com/industry-analysis/polyphenolsmarket-analysis. Accessed 07 Sept 2017

Häkkinen SH, Kärenlampi SO, Heinonen IM et al (1999) Content of the flavonols quercetin, myricetin, and kaempferol in 25 edible berries. J Agric Food Chem 47:2274-2279. doi:10.1021/JF9811065

Halvorsen BL, Carlsen MH, Phillips KM et al (2006) Content of redox-active compounds (ie, antioxidants) in foods consumed in the United States. Am J Clin Nutr 84:95-135

Hartmann U, Sagasser M, Mehrtens F et al (2005) Differential combinatorial interactions of cis-acting elements recognized by R2R3-MYB, BZIP, and BHLH factors control light-responsive and tissue-specific activation of phenylpropanoid biosynthesis genes. Plant Mol Biol 57:155-171. doi:10.1007/s11103-004-6910-0 
Hartmann A, Lemos JM, Costa RS et al (2015a) Identification of switched ARX models via convex optimization and expectation maximization. J Process Control 28:9-16. doi:10.1016/j.jprocont.2015.02.003

Hartmann A, Lemos JM, Vinga S (2015b) Modeling multiple experiments using regularized optimization: a case study on bacterial glucose utilization dynamics. Comput Biol Med 63:301-309. doi:10.1016/j.compbiomed.2014.08.027

Hartmann A, Neves AR, Lemos JM, Vinga S (2016) Identification and automatic segmentation of multiphasic cell growth using a linear hybrid model. Math Biosci 279:83-89. doi:10.1016/j.mbs.2016.06.013

Heldt H-W, Piechulla B (2011) Plant biochemistry, 4th edn. Elsevier, London

Hellström JK, Törrönen AR, Mattila PH (2009) Proanthocyanidins in common food products of plant origin. J Agric Food Chem 57:7899-7906. doi:10.1021/jf901434d

Hernández I, Molenaar D, Beekwilder J et al (2007) Expression of plant flavor genes in Lactococcus lactis. Appl Environ Microbiol 73:1544-1552. doi:10.1128/AEM.01870-06

Herrmann K, Nagel CW (1989) Occurrence and content of hydroxycinnamic and hydroxybenzoic acid compounds in foods. Crit Rev Food Sci Nutr 28:315-347. doi:10.1080/ 10408398909527504

Hujanen M, Linko S, Linko Y-Y, Leisola M (2001) Optimisation of media and cultivation conditions for $\mathrm{L}(+)(\mathrm{S})$-lactic acid production by Lactobacillus casei NRRL B-441. Appl Microbiol Biotechnol 56:126-130. doi:10.1007/s002530000501

Jain N, Ramawat KG (2013) Nutraceuticals and antioxidants in prevention of diseases. In: Ramawat KG, Merillon J-M (eds) Natural products. Springer, Berlin, pp 2559-2580

Jash SK, Mondal S (2014) Bioactive flavonoid fisetin-a molecule of pharmacological interest. Signpost Open Access J Org Biomol Chem 2:89-128

Jeandet P, Bessis R, Gautheron B (1991) The production of resveratrol $\left(3,5,4^{\prime}\right.$-trihydroxystilbene) by grape berries in different developmental stages. Am J Enol Vitic 42:41-46

Jendresen CB, Stahlhut SG, Li M et al (2015) Highly active and specific tyrosine ammonia-lyases from diverse origins enable enhanced production of aromatic compounds in bacteria and Saccharomyces cerevisiae. Appl Environ Microbiol 81:4458-4476. doi:10.1128/AEM.00405-15

Jojima T, Inui M, Yukawa H (2013) Biorefinery applications of Corynebacterium glutamicum. In: Yukawa H, Inui M (eds) Corynebacterium glutamicum: biology and biotechnology. Springer, Berlin, pp 149-172

Jones JA, Vernacchio VR, Sinkoe AL et al (2016) Experimental and computational optimization of an Escherichia coli coculture for the efficient production of flavonoids. Metab Eng 35:55-63. doi:10.1016/j.ymben.2016.01.006

Josuttis M, Verrall S, Stewart D et al (2013) Genetic and environmental effects on tannin composition in strawberry (Fragaria $\times$ ananassa) cultivars grown in different European locations. J Agric Food Chem 61:790-800. doi:10.1021/jf303725g

Ju S, Tardiff DF, Han H et al (2011) A yeast model of FUS/TLSdependent cytotoxicity. PLoS Biol 9:e1001052. doi:10. 1371/journal.pbio.1001052

Julien-Laferrière A, Bulteau L, Parrot D et al (2016) A combinatorial algorithm for microbial consortia synthetic design. Sci Rep 6:29182. doi:10.1038/srep29182
Kallscheuer N, Vogt M, Kappelmann J et al (2016a) Identification of the phd gene cluster responsible for phenylpropanoid utilization in Corynebacterium glutamicum. Appl Microbiol Biotechnol 100:1871-1881. doi:10.1007/ s00253-015-7165-1

Kallscheuer N, Vogt M, Marienhagen J (2016b) A novel synthetic pathway enables microbial production of polyphenols independent from the endogenous aromatic amino acid metabolism. ACS Synth Biol. doi:10.1021/acssynbio.6b00291

Kallscheuer N, Vogt M, Stenzel A et al (2016c) Construction of a Corynebacterium glutamicum platform strain for the production of stilbenes and (2S)-flavanones. Metab Eng 38:47-55. doi:10.1016/j.ymben.2016.06.003

Kallscheuer N, Vogt M, Bott M, Marienhagen J (2017) Functional expression of plant-derived O-methyltransferase, flavanone 3-hydroxylase, and flavonol synthase in Corynebacterium glutamicum for production of pterostilbene, kaempferol, and quercetin. J Biotechnol. doi:10.1016/j. jbiotec.2017.01.006

Kårlund A, Moor U, Sandell M, Karjalainen R (2014) The impact of harvesting, storage and processing factors on health-promoting phytochemicals in berries and fruits. Processes 2:596-624. doi:10.3390/pr2030596

Kasiotis KM, Pratsinis H, Kletsas D, Haroutounian SA (2013) Resveratrol and related stilbenes: their anti-aging and antiangiogenic properties. Food Chem Toxicol 61:112-120. doi:10.1016/j.fct.2013.03.038

Katz M, Durhuus T, Smits HP, Förster J (2011) Patent: Production of metabolites, no. WO2011147818 (A2). https:// worldwide.espacenet.com/publicationDetails/biblio?CC= WO\&NR $=2011147818$ A2 \&KC $=$ A2\&FT $=$ D. Accessed 07 Sept 2017

Kim HJ, Chang EJ, Cho SH et al (2002) Antioxidative activity of resveratrol and its derivatives isolated from seeds of Paeonia lactiflora. Biosci Biotechnol Biochem 66:1990-1993. doi:10.1271/bbb.66.1990

Koesnandar A, Nishio N, Nagai S (1990) Stimulation by cysteine on growth of Clostridium thermoaceticum in minimal medium. Appl Microbiol Biotechnol 32:711-714. doi:10. 1007/BF00164746

Kortmann M, Kuhl V, Klaffl S, Bott M (2015) A chromosomally encoded T7 RNA polymerase-dependent gene expression system for Corynebacterium glutamicum: construction and comparative evaluation at the single-cell level. Microb Biotechnol 8:253-265. doi:10.1111/1751-7915.12236

Kritzer JA, Hamamichi S, McCaffery JM et al (2009) Rapid selection of cyclic peptides that reduce $\alpha$-synuclein toxicity in yeast and animal models. Nat Chem Biol 5:655-663. doi: $10.1038 /$ nchembio. 193

Krobitsch S, Lindquist S (2000) Aggregation of huntingtin in yeast varies with the length of the polyglutamine expansion and the expression of chaperone proteins. Proc Natl Acad Sci USA 97:1589-1594. doi:10.1073/PNAS.97.4.1589

Krömer JO, Sorgenfrei O, Klopprogge K et al (2004) In-depth profiling of lysine-producing Corynebacterium glutamicum by combined analysis of the transcriptome, metabolome, and fluxome. J Bacteriol 186:1769-1784. doi:10. 1128/JB.186.6.1769-1784.2004

Kuipers OP, Beerthuyzen MM, Siezen RJ et al (1993) Characterization of the nisin gene cluster nisABTCIPR of Lactococcus lactis. Requirement of expression of the nisA and 
nisI genes for development of immunity. Eur J Biochem 216:281-291. doi:10.1111/j.1432-1033.1993.tb18143.x

Kuipers OP, de Ruyter PGG, Kleerebezem M, de Vos WM (1998) Quorum sensing-controlled gene expression in lactic acid bacteria. J Biotechnol 64:15-21. doi:10.1016/ S0168-1656(98)00100-X

Kula M, Majdan M, Głód D, Krauze-Baranowska M (2016) Phenolic composition of fruits from different cultivars of red and black raspberries grown in Poland. J Food Compos Anal 52:74-82. doi:10.1016/j.jfca.2016.08.003

Kunji ERS, Chan KW, Slotboom DJ et al (2005) Eukaryotic membrane protein overproduction in Lactococcus lactis. Curr Opin Biotechnol 16:546-551. doi:10.1016/j.copbio. 2005.08.006

Kwiatkowski TJ, Bosco DA, LeClerc AL et al (2009) Mutations in the FUS/TLS gene on chromosome 16 cause familial amyotrophic lateral sclerosis. Science 323:1205-1208. doi:10.1126/science. 1166066

Lahtinen S, Ouwehand AC, Salminen S, von Wright A (eds) (2011) Lactic acid bacteria: microbiological and functional aspects, 4th edn. CRC Press, Boca Raton

Landete JM (2011) Ellagitannins, ellagic acid and their derived metabolites: a review about source, metabolism, functions and health. Food Res Int 44:1150-1160. doi:10.1016/j. foodres.2011.04.027

Lenihan JR, Tsuruta H, Diola D et al (2008) Developing an industrial artemisinic acid fermentation process to support the cost-effective production of antimalarial artemisininbased combination therapies. Biotechnol Prog 24:1026-1032. doi:10.1002/btpr.27

Li H, Qiu T, Huang G et al (2010) Production of gammaaminobutyric acid by Lactobacillus brevis NCL912 using fed-batch fermentation. Microb Cell Fact 9:85. doi:10. 1186/1475-2859-9-85

Li X, Sun H, Pei J et al (2012) De novo sequencing and comparative analysis of the blueberry transcriptome to discover putative genes related to antioxidants. Gene 511:54-61. doi:10.1016/j.gene.2012.09.021

Linares DM, Kok J, Poolman B (2010) Genome sequences of Lactococcus lactis MG1363 (revised) and NZ9000 and comparative physiological studies. J Bacteriol 192:5806-5812. doi:10.1128/JB.00533-10

Liu Y-B, Long M-X, Yin Y-J et al (2013) Physiological roles of mycothiol in detoxification and tolerance to multiple poisonous chemicals in Corynebacterium glutamicum. Arch Microbiol 195:419-429. doi:10.1007/s00203-013-0889-3

Logan BE, Rabaey K (2012) Conversion of wastes into bioelectricity and chemicals by using microbial electrochemical technologies. Science 337:686-690. doi:10. 1126/science. 1217412

Luo J, Nishiyama Y, Fuell C et al (2007) Convergent evolution in the BAHD family of acyl transferases: identification and characterization of anthocyanin acyl transferases from Arabidopsis thaliana. Plant J 50:678-695. doi:10.1111/j. 1365-313X.2007.03079.x

Määttä KR, Kamal-Eldin A, Törrönen R (2001) Phenolic compounds in berries of black, red, green, and white currants (Ribes sp.). Antioxid Redox Signal 3:981-993. doi:10.1089/152308601317203521

Määttä KR, Kamal-Eldin A, Törrönen AR (2003) High-performance liquid chromatography (HPLC) analysis of phenolic compounds in berries with diode array and electrospray ionization mass spectrometric (MS) detection: Ribes species. J Agric Food Chem 51:6736-6744. doi:10. 1021/JF0347517

MacDonald MJ, D'Cunha GB (2007) A modern view of phenylalanine ammonia lyase. Biochem Cell Biol 85:273-282. doi:10.1139/O07-018

Machado D, Herrgård MJ, Rocha I (2015) Modeling the contribution of allosteric regulation for flux control in the central carbon metabolism of E. coli. Front Bioeng Biotechnol 3:154. doi:10.3389/fbioe.2015.00154

Maeda S, Gasson MJ (1986) Cloning, expression and location of the Streptococcus lactis gene for phospho- $\beta$-d-galactosidase. Microbiology 132:331-340. doi:10.1099/00221287132-2-331

Maher P (2009) Modulation of multiple pathways involved in the maintenance of neuronal function during aging by fisetin. Genes Nutr 4:297-307. doi:10.1007/s12263-0090142-5

Maher P, Dargusch R, Ehren JL et al (2011) Fisetin lowers methylglyoxal dependent protein glycation and limits the complications of diabetes. PLoS ONE 6:e21226. doi:10. 1371/journal.pone.0021226

Manganaris GA, Goulas V, Vicente AR, Terry LA (2014) Berry antioxidants: small fruits providing large benefits. J Sci Food Agric 94:825-833. doi:10.1002/jsfa.6432

Marhuenda J, Alemán MD, Gironés-Vilaplana A et al (2016) Phenolic composition, antioxidant activity, and in vitro availability of four different berries. J Chem 2016:1-7. doi:10.1155/2016/5194901

Marienhagen J, Bott M (2013) Metabolic engineering of microorganisms for the synthesis of plant natural products. J Biotechnol 163:166-178. doi:10.1016/j.jbiotec.2012.06. 001

Mazur SP, Sønsteby A, Nes A et al (2014) Effects of postflowering environmental variation along an altitudinal gradient on chemical composition of "Glen Ample" Red Raspberry (Rubus idaeus L.). Eur J Hortic Sci 79:267-277

McDougall G, Martinussen I, Stewart D (2008) Towards fruitful metabolomics: high throughput analyses of polyphenol composition in berries using direct infusion mass spectrometry. J Chromatogr B 871:362-369. doi:10.1016/j. jchromb.2008.06.032

Menezes R, Tenreiro S, Macedo D et al (2015) From the baker to the bedside: yeast models of Parkinson's disease. Microb Cell 2:262-279. doi:10.15698/mic2015.08.219

Miladinović B, Kostić M, Šavikin K et al (2014) Chemical profile and antioxidative and antimicrobial activity of juices and extracts of 4 black currants varieties (Ribes nigrum L.). J Food Sci 79:C301-C309. doi:10.1111/1750-3841.12364

Milivojević J, Maksimović V, Nikolić M et al (2011) Chemical and antioxidant properties of cultivated and wild Fragaria and Rubus berries. J Food Qual 34:1-9. doi:10.1111/j. 1745-4557.2010.00360.x

Miller-Fleming L, Giorgini F, Outeiro TF (2008) Yeast as a model for studying human neurodegenerative disorders. Biotechnol J 3:325-338. doi:10.1002/biot.200700217

Milreu PV, Klein CC, Cottret L et al (2014) Telling metabolic stories to explore metabolomics data: a case study on the yeast response to cadmium exposure. Bioinformatics 30:61-70. doi:10.1093/bioinformatics/btt597 
Morgera E, Tsioumani E, Buck M (2014) Unraveling the Nagoya protocol. Brill, Leiden

Moulton GG (2014) Fed-batch fermentation. Woodhead Publishing, Cambridge

Moyer RA, Hummer KE, Finn CE et al (2001) Anthocyanins, phenolics, and antioxidant capacity in diverse small fruits: vaccinium, Rubus, and Ribes. J Agric Food Chem 50:519-525. doi:10.1021/JF011062R

Neves AR, Pool WA, Kok J et al (2005) Overview on sugar metabolism and its control in Lactococcus lactis-the input from in vivo NMR. FEMS Microbiol Rev 29:531-554. doi:10.1016/j.fmrre.2005.04.005

NMRO (UK) (2015) The Nagoya protocol on access and benefit sharing of genetic resources: compliance and guidance (ABS). https://www.gov.uk/guidance/abs. Accessed 07 Sept 2017

O'Brien RJ, Wong PC (2011) Amyloid precursor protein processing and Alzheimer's disease. Ann Rev Neurosci 34:185-204. doi:10.1146/annurev-neuro-061010-113613

Oliveira AP, Nielsen J, Förster J (2005) Modeling Lactococcus lactis using a genome-scale flux model. BMC Microbiol 5:39. doi:10.1186/1471-2180-5-39

Outeiro TF, Lindquist S (2003) Yeast cells provide insight into alpha-synuclein biology and pathobiology. Science 302:1772-1775. doi:10.1126/science.1090439

Palmieri N, Forleo MB, Salimei E (2017) Environmental impacts of a dairy cheese chain including whey feeding: an Italian case study. J Clean Prod 140:881-889. doi:10.1016/ j.jclepro.2016.06.185

Pan M-G, Xiong Y, Chen F (2013) NFAT gene family in inflammation and cancer. Curr Mol Med 13:543-554

Pandey RP, Parajuli P, Koffas MAG, Sohng JK (2016) Microbial production of natural and non-natural flavonoids: pathway engineering, directed evolution and systems/ synthetic biology. Biotechnol Adv 34:634-662. doi:10. 1016/j.biotechadv.2016.02.012

Paredes-López O, Cervantes-Ceja ML, Vigna-Pérez M, Hernández-Pérez TT (2010) Berries: improving human health and healthy aging, and promoting quality life — a review. Plant Foods Human Nutr 65:299-308. doi:10.1007/ s11130-010-0177-1

Pátek M, Nešvera J (2013) Promoters and plasmid vectors of Corynebacterium glutamicum. In: Yukawa H, Inui M (eds) Corynebacterium glutamicum: biology and biotechnology, 23rd edn. Springer, Berlin, pp 51-88

Patil K, Rocha I, Förster J et al (2005) Evolutionary programming as a platform for in silico metabolic engineering. BMC Bioinform 6:308. doi:10.1186/1471-2105-6-308

Pauli GF, Chen S-N, Friesen JB et al (2012) Analysis and purification of bioactive natural products: the AnaPurNa study. J Nat Prod 75:1243-1255. doi:10.1021/np300066q

Pedersen MB, Gaudu P, Lechardeur D et al (2012) Aerobic respiration metabolism in lactic acid bacteria and uses in biotechnology. Ann Rev Food Sci Technol 3:37-58. doi:10.1146/annurev-food-022811-101255

Pei L, Schmidt M (2016) Sustainable assessment on using bacterial platform to produce high-added-value products from berries through metabolic engineering. In: Singh S (ed) Systems biology application in synthetic biology. Springer, New Delhi, pp 71-78

Pontes DS, de Azevedo MSP, Chatel J-M et al (2011) Lactococcus lactis as a live vector: heterologous protein production and DNA delivery systems. Protein Expr Purif 79:165-175. doi:10.1016/j.pep.2011.06.005

Prescott TAK, Ariño J, Kite GC, Simmonds MSJ (2012) Inhibition of human calcineurin and yeast calcineurin-dependent gene expression by Jasminum humile leaf and root extracts. J Ethnopharmacol 140:293-297. doi:10.1016/j. jep.2012.01.020

Prince K, Smith M (2004) Protein purification protocols. In: Cutler P (ed) Protein purification protocols. Humana Press, Totowa, pp 463-480

Quideau S, Deffieux D, Douat-Casassus C, Pouységu L (2011) Plant polyphenols: chemical properties, biological activities, and synthesis. Angew Chem Int Ed 50:586-621. doi:10.1002/anie.201000044

Rani MU, Appaiah A (2011) Optimization of culture conditions for bacterial cellulose production from Gluconacetobacter hansenii UAC09. Ann Microbiol 61:781-787. doi:10. 1007/s13213-011-0196-7

Rasmussen SE, Frederiksen H, Struntze Krogholm K, Poulsen L (2005) Dietary proanthocyanidins: occurrence, dietary intake, bioavailability, and protection against cardiovascular disease. Mol Nutr Food Res 49:159-174. doi:10. 1002/mnfr.200400082

Ratnam BVV, Narasimha Rao M, Damodar Rao M et al (2003) Optimization of fermentation conditions for the production of ethanol from sago starch using response surface methodology. World J Microbiol Biotechnol 19:523-526. doi:10.1023/A:1025174731814

Renaud S, de Lorgeril M (1992) Wine, alcohol, platelets, and the French paradox for coronary heart disease. Lancet 339:1523-1526. doi:10.1016/0140-6736(92)91277-F

Ro D-K, Paradise EM, Ouellet M et al (2006) Production of the antimalarial drug precursor artemisinic acid in engineered yeast. Nature 440:940-943. doi:10.1038/nature04640

Ruiz A, Hermosín-Gutiérrez I, Mardones C et al (2010) Polyphenols and antioxidant activity of calafate (Berberis microphylla) fruits and other native berries from Southern Chile. J Agric Food Chem 58:6081-6089. doi:10.1021/ jf100173x

Rusnak F, Mertz P (2000) Calcineurin: form and function. Physiol Rev 80:1483-1521

Santos CNS, Koffas M, Stephanopoulos G (2011) Optimization of a heterologous pathway for the production of flavonoids from glucose. Metab Eng 13:392-400. doi:10.1016/j. ymben.2011.02.002

SCBD (2011) Nagoya protocol on access to genetic resources and the fair and equitable sharing of benefits arising from their utilization to the convention on biological diversity. https://www.cbd.int/abs/doc/protocol/nagoya-protocol-en. pdf. Accessed 07 Sept 2017

Secretary of State (UK) (2015) Nagoya protocol (compliance) regulations 2015. http://www.legislation.gov.uk/uksi/ 2015/821/pdfs/uksi_20150821_en.pdf. Accessed 07 Sept 2017

Segrè D, Vitkup D, Church GM (2002) Analysis of optimality in natural and perturbed metabolic networks. Proc Natl Acad Sci USA 99:15112-15117. doi:10.1073/pnas.232349399

Shen X, Liu S (2005) Key enzymes of the protocatechuate branch of the $\beta$-ketoadipate pathway for aromatic degradation in Corynebacterium glutamicum. Sci China Ser C Life Sci 48:241-249. doi:10.1007/BF03183617 
Shen X-H, Zhou N-Y, Liu S-J (2012) Degradation and assimilation of aromatic compounds by Corynebacterium glutamicum: another potential for applications for this bacterium? Appl Microbiol Biotechnol 95:77-89. doi:10. 1007/s00253-012-4139-4

Shinfuku Y, Sorpitiporn N, Sono M et al (2009) Development and experimental verification of a genome-scale metabolic model for Corynebacterium glutamicum. Microb Cell Fact 8:43. doi:10.1186/1475-2859-8-43

Shults CW (2006) Lewy bodies. Proc Natl Acad Sci USA 103:1661-1668. doi:10.1073/pnas.0509567103

Simeonidis E, Price ND (2015) Genome-scale modeling for metabolic engineering. J Ind Microbiol Biotechnol 42:327-338. doi:10.1007/s10295-014-1576-3

Song AA, Abdullah JO, Abdullah MP et al (2014) Engineering the lactococcal mevalonate pathway for increased sesquiterpene production. FEMS Microbiol Lett 355:177-184. doi:10.1111/1574-6968.12469

Stahlhut SG, Siedler S, Malla S et al (2015) Assembly of a novel biosynthetic pathway for production of the plant flavonoid fisetin in Escherichia coli. Metab Eng 31:84-93. doi:10. 1016/j.ymben.2015.07.002

Stanford NJ, Millard P, Swainston N (2015) RobOKoD: microbial strain design for (over)production of target compounds. Front Cell Dev Biol 3:17. doi:10.3389/fcell. 2015.00017

Stavang JA, Freitag S, Foito A et al (2015) Raspberry fruit quality changes during ripening and storage as assessed by colour, sensory evaluation and chemical analyses. Sci Hortic (Amst) 195:216-225. doi:10.1016/j.scienta.2015. 08.045

Stracke R, Werber M, Weisshaar B (2001) The R2R3-MYB gene family in Arabidopsis thaliana. Curr Opin Plant Biol 4:447-456. doi:10.1016/S1369-5266(00)00199-0

Su LJ, Auluck PK, Outeiro TF et al (2010) Compounds from an unbiased chemical screen reverse both ER-to-Golgi trafficking defects and mitochondrial dysfunction in Parkinson's disease models. Dis Model Mech 3:194-208. doi:10. $1242 / \mathrm{dmm} .004267$

Suh Y, Afaq F, Johnson JJ, Mukhtar H (2008) A plant flavonoid fisetin induces apoptosis in colon cancer cells by inhibition of COX2 and Wnt/EGFR/NF-B-signaling pathways. Carcinogenesis 30:300-307. doi:10.1093/carcin/bgn269

Tardiff DF, Tucci ML, Caldwell KA et al (2012) Different 8 -hydroxyquinolines protect models of TDP-43 protein, $\alpha$ synuclein, and polyglutamine proteotoxicity through distinct mechanisms. J Biol Chem 287:4107-4120. doi:10. 1074/jbc.M111.308668

Tayarani-Najaran Z, Mousavi SH, Tajfard F et al (2013) Cytotoxic and apoptogenic properties of three isolated diterpenoids from Salvia chorassanica through bioassayguided fractionation. Food Chem Toxicol 57:346-351. doi:10.1016/j.fct.2013.03.037

Tepper N, Shlomi T (2010) Predicting metabolic engineering knockout strategies for chemical production: accounting for competing pathways. Bioinformatics 26:536-543. doi:10.1093/bioinformatics/btp704

Tohge T, Nishiyama Y, Hirai MY et al (2005) Functional genomics by integrated analysis of metabolome and transcriptome of Arabidopsis plants over-expressing an MYB transcription factor. Plant J 42:218-235. doi:10.1111/j. 1365-313X.2005.02371.X

Törrönen R (2009) Sources and health effects of dietary ellagitannins. In: Quideau S (ed) Chemistry and biology of ellagitannins: an underestimated class of bioactive plant polyphenols. World Scientific Publishing, Singapore, pp 298-319

Vagiri M, Ekholm A, Öberg E et al (2013) Phenols and ascorbic acid in black currants (Ribes nigrum L.): variation due to genotype, location, and year. J Agric Food Chem 61:9298-9306. doi:10.1021/jf402891s

van Summeren-Wesenhagen PV, Marienhagen J (2015) Metabolic engineering of Escherichia coli for the synthesis of the plant polyphenol pinosylvin. Appl Environ Microbiol 81:840-849. doi:10.1128/AEM.02966-14

Vance C, Rogelj B, Hortobágyi T et al (2009) Mutations in FUS, an RNA processing protein, cause familial amyotrophic lateral sclerosis type 6. Science 323:1208-1211. doi:10. 1126/science. 1165942

Vazquez-Albacete D, Cavaleiro AM, Christensen U et al (2016) An expression tag toolbox for microbial production of membrane bound plant cytochromes P450. Biotechnol Bioeng. doi:10.1002/bit.26203

Vera J, González-Alcón C, Marín-Sanguino A, Torres N (2010) Optimization of biochemical systems through mathematical programming: methods and applications. Comput Oper Res 37:1427-1438. doi:10.1016/j.cor.2009.02.021

Veríssimo A, Bassard J-E, Julien-Laferrière A, et al. (2017) MassBlast: A workflow to accelerate RNA-seq and DNA database analysis. bioRxiv 131953. doi:10.1101/131953

Vogt T (2010) Phenylpropanoid biosynthesis. Mol Plant 3:2-20. doi: $10.1093 / \mathrm{mp} / \mathrm{ssp} 106$

Waldo GS, Standish BM, Berendzen J, Terwilliger TC (1999) Rapid protein-folding assay using green fluorescent protein. Nat Biotechnol 17:691-695. doi:10.1038/10904

Wang Y, Bhuiya MW, Zhou R, Yu O (2015) Pterostilbene production by microorganisms expressing resveratrol O-methyltransferase. Ann Microbiol 65:817-826. doi:10. 1007/s13213-014-0922-Z

Wang J, Guleria S, Koffas MA, Yan Y (2016a) Microbial production of value-added nutraceuticals. Curr Opin Biotechnol 37:97-104. doi:10.1016/j.copbio.2015.11.003

Wang Y, Zhu J, Meng X et al (2016b) Comparison of polyphenol, anthocyanin and antioxidant capacity in four varieties of Lonicera caerulea berry extracts. Food Chem 197:522-529. doi:10.1016/j.foodchem.2015.11.006

Watts KR, Ratnam J, Ang K-H et al (2010) Assessing the trypanocidal potential of natural and semi-synthetic diketopiperazines from two deep water marine-derived fungi. Bioorganic Med Chem 18:2566-2574. doi:10.1016/j.bmc. 2010.02.034

Weckwerth W (2003) Metabolomics in systems biology. Ann Rev Plant Biol 54:669-689. doi:10.1146/annurev.arplant. 54.031902.135014

Weller MG (2012) A unifying review of bioassay-guided fractionation, effect-directed analysis and related techniques. Sensors 12:9181-9209. doi:10.3390/s120709181

Wen Z, Minton NP, Zhang Y et al (2016) Enhanced solvent production by metabolic engineering of a twin-clostridial consortium. Metab Eng. doi:10.1016/j.ymben.2016.10.013

Xu P, Ranganathan S, Fowler ZL et al (2011) Genome-scale metabolic network modeling results in minimal 
interventions that cooperatively force carbon flux towards malonyl-CoA. Metab Eng 13:578-587. doi:10.1016/j. ymben.2011.06.008

Xu P, Wang W, Li L et al (2013) Design and kinetic analysis of a hybrid promoter-regulator system for malonyl-CoA sensing in Escherichia coli. ACS Chem Biol. doi:10.1021/ cb400623m

Yang X, Summerhurst DK, Koval SF et al (2001) Isolation of an antimicrobial compound from Impatiens balsamina $\mathrm{L}$. using bioassay-guided fractionation. Phytother Res 15:676-680. doi:10.1002/ptr.906

Yu C, Cao Y, Zou H, Xian M (2011) Metabolic engineering of Escherichia coli for biotechnological production of highvalue organic acids and alcohols. Appl Microbiol Biotechnol 89:573-583. doi:10.1007/s00253-010-2970-Z

Zadernowski R, Naczk M, Nesterowicz J (2005) Phenolic acid profiles in some small berries. J Agric Food Chem 53:2118-2124. doi:10.1021/JF040411P
Zelle RM, de Hulster E, Kloezen W et al (2010) Key process conditions for production of $\mathrm{C}(4)$ dicarboxylic acids in bioreactor batch cultures of an engineered Saccharomyces cerevisiae strain. Appl Environ Microbiol 76:744-750. doi:10.1128/AEM.02396-09

Zhang H, Matsuda H, Yamashita C et al (2009) Hydrangeic acid from the processed leaves of Hydrangea macrophylla var. thunbergii as a new type of anti-diabetic compound. Eur J Pharmacol 606:255-261. doi:10.1016/j.ejphar.2009.01.005

Zheng J, Ding C, Wang L et al (2011) Anthocyanins composition and antioxidant activity of wild Lycium ruthenicum Murr. from Qinghai-Tibet Plateau. Food Chem 126:859-865. doi:10.1016/j.foodchem.2010.11.052

Zomorrodi AR, Suthers PF, Ranganathan S, Maranas CD (2012) Mathematical optimization applications in metabolic networks. Metab Eng 14:672-686. doi:10.1016/j.ymben. 2012.09.005 